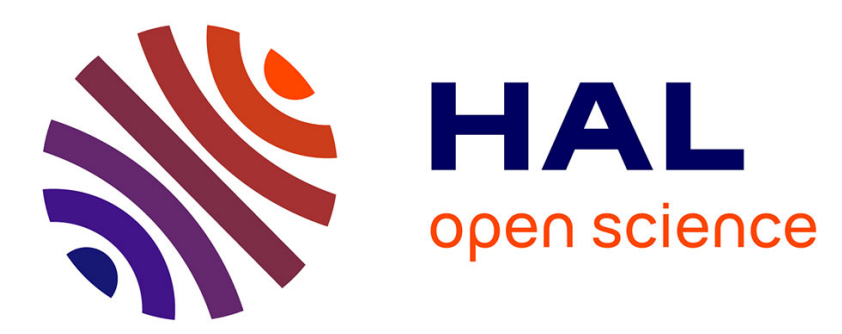

\title{
Dynamique de la rivière Poulvar et morphogenèse de la plaine de Tang-i Bulaghi (Fars, Iran) à l'Holocène. Premiers résultats
}

Jean-Baptiste Rigot

\section{- To cite this version: \\ Jean-Baptiste Rigot. Dynamique de la rivière Poulvar et morphogenèse de la plaine de Tang-i Bulaghi (Fars, Iran) à l'Holocène. Premiers résultats. Géomorphologie : relief, processus, environnement, 2010, pp.57-72. hal-00943455}

\section{HAL Id: hal-00943455 \\ https://hal.science/hal-00943455}

Submitted on 7 Feb 2014

HAL is a multi-disciplinary open access archive for the deposit and dissemination of scientific research documents, whether they are published or not. The documents may come from teaching and research institutions in France or abroad, or from public or private research centers.
L'archive ouverte pluridisciplinaire HAL, est destinée au dépôt et à la diffusion de documents scientifiques de niveau recherche, publiés ou non, émanant des établissements d'enseignement et de recherche français ou étrangers, des laboratoires publics ou privés. 
$1 / 2010(2010)$

Varia

Jean-Baptiste Rigot

\title{
Dynamique de la rivière Poulvar et morphogenèse de la plaine de Tang- i Bulaghi (Fars, Iran) à l'Holocène. Premiers résultats
}

\begin{abstract}
Avertissement
Le contenu de ce site relève de la législation française sur la propriété intellectuelle et est la propriété exclusive de l'éditeur.

Les œuvres figurant sur ce site peuvent être consultées et reproduites sur un support papier ou numérique sous réserve qu'elles soient strictement réservées à un usage soit personnel, soit scientifique ou pédagogique excluant toute exploitation commerciale. La reproduction devra obligatoirement mentionner l'éditeur, le nom de la revue, l'auteur et la référence du document.

Toute autre reproduction est interdite sauf accord préalable de l'éditeur, en dehors des cas prévus par la législation en vigueur en France.
\end{abstract}

\section{revues.org}

Revues.org est un portail de revues en sciences humaines et sociales développé par le Cléo, Centre pour l'édition électronique ouverte (CNRS, EHESS, UP, UAPV).

Référence électronique

Jean-Baptiste Rigot, « Dynamique de la rivière Poulvar et morphogenèse de la plaine de Tang-i Bulaghi (Fars, Iran) à l'Holocène. Premiers résultats », Géomorphologie : relief, processus, environnement [En ligne], 1/2010 | 2010, mis en ligne le 01 avril 2012, consulté le 15 octobre 2012. URL : http://geomorphologie.revues.org/7813 ; DOI : 10.4000/geomorphologie.7813

Éditeur : Groupe français de géomorphologie http://geomorphologie.revues.org http://www.revues.org

Document accessible en ligne sur : http://geomorphologie.revues.org/7813 Ce document est le fac-similé de l'édition papier.

(c) Groupe français de géomorphologie 


\title{
Dynamique de la rivière Poulvar et morphogenèse de la plaine de Tang-i Bulaghi (Fars, Iran) à l'Holocène. Premiers résultats
}

\section{Dynamics of the Poulvar River and morphological history of the Tang-i Bulaghi plain (Fars, Iran) during the Holocene. First results}

\author{
Jean-Baptiste Rigot*
}

\begin{abstract}
Résumé
L'étude géomorphologique d'une partie du bassin-versant du Poulvar (Fars, Iran) a mis en évidence une série de nappes alluviales holocènes. La plus remarquable forme une terrasse de $17 \mathrm{~m}$ d'épaisseur. Constituée principalement de limons, cette formation s'est mise en place durant l'Holocène ancien et moyen. Une telle formation est inattendue dans le milieu montagnard régional. L'étude montre que le cadre structural et les forçages climatiques en sont probablement à l'origine. D'une part, cette sédimentation est intervenue dans le contexte relativement plus humide de l'Optimum Climatique Holocène, qui a favorisé le décapage des sols lœssiques de versants. D'autre part, les plaines d'origine structurale, situées à l'aval du bassin-versant, expliquent le haut niveau de base du cours d'eau, sa faible pente longitudinale et la tendance à l'aggradation. À cet épisode fait suite une longue période dominée par une dynamique d'incision, interrompue par deux phases de sédimentation. Les données sont encore limitées pour expliquer cette évolution holocène qui a probablement une double origine : le retour de l'aridité autour de 6000 BP et l'exhaussement du Zagros.
\end{abstract}

Mots clés : Holocène, morphostructure, dynamique fluviale, limons, aridité, Iran.

\begin{abstract}
The geomorphologic study of a part of the Poulvar River basin (Fars, Iran) has brought to light a series of Holocene deposits. The main deposit is $17 \mathrm{~m}$ thick, consisting mainly of silty sediments deposited during the early and middle Holocene. These deposits are unusual in such a mountainous environment. The study shows that structural and climate factors could explain this sedimentation. On the one hand, it occurred during the relatively humid Holocene Climatic Optimum, which may have contributed to soil erosion. On the other hand, the plains of structural origin located downstream from the Poulvar basin would explain the high base level of the river, the low incline of the longitudinal profile and the tendency towards sedimentation. This major episode is followed by a long period of erosion with only two phases of sedimentation. Data are still lacking to explain this phenomenon, which probably had two major causes: the return of aridity at about $6000 \mathrm{BP}$ and the Zagros uplift.
\end{abstract}

Key words: Holocene, morphostructure, fluvial dynamics, erosion, silt, aridity, Iran.

\section{Abridged English version}

The study focuses on a region for which there is little geomorphological data. The Poulvar valley and the Tang-i Bulaghi plain are situated in the Fars region, in south-central Iran (fig. 1A). They are part of the southern Zagros Mountains, which formed as a result of the collision of the Arabian and Eurasian tectonic plates. The structural geometry accounts for the drainage morphology that was first organi- sed along the fold in a NW-SE direction, and secondarily in a NE-SW direction, by superimposition (fig. 1B). The Poulvar River belongs to one of these secondary sections (fig. 1C). The climate is a dry Mediterranean type, characterised by winter rainfall and long dry summers (fig. 5). The Tang-i Bulaghi plain is situated in a syncline of Cretaceous limestone (fig. 2). To the east it is bordered by a wrench fault occupied by the Poulvar River (fig. 2). The valley is 150 to $300 \mathrm{~m}$ wide and is as wide as $600 \mathrm{~m}$ wide where it enters the Tang-i Bu-

\footnotetext{
* Université François-Rabelais, CITERES-UMR 6173, Laboratoire Archéologie et Territoires, 35, allée Ferdinand de Lesseps, BP 60449, 37204 Tours Cedex 03. Courriel : jb.rigot@univ-tours.fr
} 
laghi plain. It has a complex history, with alternating deposition and erosion phases. This paper presents the initial results of the geomorphological study, and focuses on the deposition phases and the sediments. The study of the terraces and the sediments provides an incomplete but fresh picture of the Holocene history of the valley (the sections can be seen in fig. 3). Three Holocene fill terraces can be observed in the valley; the oldest one (T1) is $17 \mathrm{~m}$ thick and consists of silt and sand sediments (fig. 3 and fig. 4); the second fill terrace is $6 \mathrm{~m}$ thick and consists of heterometric material in a silty matrix; the last one is a $3 \mathrm{~m}$ thick fill terrace made of coarse material. Five ${ }^{14} \mathrm{C}$ dates on charcoal were carried out in the Poulvar valley and in the Persepolis plain (tab. 1). They give a date of $7945 \pm 50 \mathrm{BP}(\mathrm{Ly}$-3364) for the lower part of the T1 terrace and $1758 \pm 45 B P$ (LTL4130A) at the top of the same terrace (T1); $1243 \pm 45$ BP (LTL4127A) and $990 \pm 35 B P(L y-3365)$ for the second terrace (T2); $140 \pm$ $30 \mathrm{BP}$ (Ly-3363) for the most recent terrace (T3). The Tang-i Bulaghi plain is located in a syncline, bounded to the north by an anticline. The escarpment reaches $600 \mathrm{~m}$ to the north and $200 \mathrm{~m}$ to the south of the plain. This morphology is responsible for the occurrence of alluvial fans in slope foot positions at the edge of the plain. The granulation of the deposits is mixed, with a high percentage of coarse material.

Our first results show that after a strong erosion phase during the Post-Glacial Period, a deposition phase began, probably around 9000-8000 BP, during the moister and warmer Holocene Climatic Optimum (HCO). This event has been reported for neighbouring regions and for the Persian Gulf(Uchupi et al., 1999; Kehl et al., 2005). The mostly silty grain size distribution of the sediments and the thickness of the terraces are unusual in the mountainous environment of the Poulvar. Pebbles and cobbles should be expected at this altitude $(1800 \mathrm{~m})$, whereas silt and small-grained sand should have been transferred. Moreover, the increase of humidity during the $\mathrm{HCO}$ would lead to the erosion of the valley. On the one hand, we presume that rainfall probably remained low and that vegetation was still steppic during this period (Miller and Kimiaie, 2006). These conditions would not have led to high erosion in river channels. On the other hand, the silty sediment deposited in the channel comes from the loess like outwash deposition and soil erosion. We believe that this dynamic has a morphological and structural origin. The downstream plains (fig. 1C) account for the low incline of the longitudinal profile of the stream and its high base level. The consequences are a slow discharge downstream and sedimentation upstream. This episode is followed by strong erosion in the Poulvar channel. Though new ${ }^{14} \mathrm{C}$ dates are available for the terrace $\mathrm{T} 2$, we expect more carbon dating to define the chronology and the duration of this event. It could have been caused by the slow sediment accumulation and the return of a drier climate. Neotectonics may also have played a role in this dynamic because of the collisional geometry of the Zagros. The Arabian plate is presently moving northward towards Eurasia at a rate of $22 \pm 2 \mathrm{~mm} / \mathrm{a}$, and a part of this convergence, around $7 \mathrm{~mm} / \mathrm{a}$, is taken up by the Zagros (Vernant, 2003).
A similar movement has been evaluated for the last 5000$6000 \mathrm{ka}$ (Blanc et al., 2003). We can assume that this would have been the case during the Holocene. A phase of alluvial aggradation followed, which is not clearly dated. According to the ${ }^{14} \mathrm{C}$ date obtained on a charcoal sample in the two top meters of the high terrace, this aggradation would have taken place after $1750 \mathrm{BP}$, and before $1000 \mathrm{BP}$, as $3.5 \mathrm{~m}$ of the fill terrace $T 2$ were already deposited. But this time period seems too short and we think that this top deposit may be a more recent colluvial deposit. The deposition phase was probably induced by two proxy variables: an agricultural activity responsible for the soil erosion along the slope and a moister climate. Finding palaeoenvironmental evidence for the latter event is difficult, because it may be a simple change in the rainfall distribution during the year. This episode is followed by two phases of erosion and sedimentation in the valley. These erosion phases, which are not precisely situated in time, may have been partly caused by a new equilibrium of the fluvial system in relation to another variation in rainfall and to human migration. The sedimentation is recent and consists of coarse material. The last fill terrace is a few meters thick and is a part of the braided channel that occurs during the Little Ice Age. The geomorphological history of the Tang-i Bulaghi plain is difficult to describe at the present time because of a lack of dating. But the archaeological remains found on the top layer of the alluvial fans, from the Bakun period (5000 BC) to the Islamic period (AD 636; Atai and Boucharlat, 2009; Helwing and Seyedin, 2009; fig. 6), suggest limited morphogenetic activity during the middle and late Holocene.

These initial results clarify the dynamics of the river and the morphological history of the plain during the early and middle Holocene. The structural plains located downstream from the Poulvar basin are mainly responsible for the sedimentation of a thick and silty deposits in the Poulvar valley. Brief episodes of sedimentation followed, but the main geomorphic activity during the late Holocene is channel incision, as suggested by the thickness of the lower Holocene fill terrace. Low rates of sedimentation are reported in the Tang$i$ Bulaghi plain, where archaeological remains have been found on the slope and on the top of the alluvial fans. Erosion is also very soft after the lower Holocene. This dynamic may have been partly caused by aridity after $6000 \mathrm{BP}(\mathrm{Re}$ gard et al., 2006) and partly by neotectonism. We still expect to find evidences for the latter phenomenon.

\section{Introduction}

Cet article présente les questions soulevées par l'étude géomorphologique de la région de Pasargades-Tang-i Bulaghi et les premiers résultats obtenus. L'objectif de cette recherche est de contribuer à la connaissance de l'évolution du paysage montagnard à l'Holocène dans l'Iran semi-aride et plus largement, dans les régions proche-orientales continentales en domaine semi-aride. Dans ce cadre, l'étude fait suite à d'autres travaux de géomorphologie réalisés en Iran ces dernières années (Kehl et al., 2005 ; Djamali et al., 2006 ; Regard et al., 2006 ; Fouache, 2007 ; Kehl et al., 2009). 
L'intérêt du travail est double : d'une part il concerne un secteur d'étude, le Zagros, en Iran, dont l'évolution géomorphologique à l'Holocène est encore assez mal connue ; d'autre part, il profite d'une région dont le cadre structural permet le piégeage de grands volumes de sédiments fins et facilite l'étude des archives sédimentaires sur le temps long.

L'évolution du paysage est appréhendée à l'aune des différents forçages, en particulier climatiques et anthropiques, au moment où, dans ces régions, le réchauffement post-glaciaire et le début de la mise en valeur agricole créent des conditions particulièrement morphogènes. L'interprétation climatique de la morphogenèse repose sur les notions générales de stabilité et d'instabilité du paysage, dérivées de la théorie de la bio-rhexistasie (Erhart, 1956 ; Tricart, 1977). On distingue des phases stables, pendant lesquelles la morphogenèse est limitée et un sol peut se développer et des phases instables, géomorphologiquement actives. L'idée est admise que ces phases sont conditionnées en grande partie par la densité du couvert végétal, directement dépendante du climat. En période plus sèche, le taux de recouvrement de la végétation est faible, ce qui entraîne une activité érosive forte sur les versants. En période plus humide, le taux de recouvrement végétal s'accroît et l'érosion sur les versants diminue. Cependant, ce schéma est à nuancer en domaine semi-aride et plus encore en domaine aride (Coque, 1998). En effet, dans ce cas, le temps de réponse de la végétation à une modification du climat est beaucoup plus lent. Ainsi, nous avons constaté, dans les marges arides du nord de la Syrie (Rigot, 2003 et 2006), que c'est dans un contexte d'accroissement des précipitations, dans un milieu qui reste à dominante sèche, que se produit l'essentiel de l'érosion puis du dépôt. C'est un constat qui est fait ailleurs au Proche-Orient ou en domaine méditerranéen même si, dans bien des cas, l'activité humaine accentue le processus (Neboit, 1991 ; Sanlaville, 1992 ; Courty, 1994 ; Wengler et al., 1994 ; Wengler, 1995 ; Geyer et Besançon, 1997 ; Ballais et al., 2003 ; Weisrock et al., 2006 ; Bkhairi et Karray, 2008). Plus généralement, il semble qu'en milieu sec, les crises érosives interviennent au moment des transitions climatiques, quel qu'en soit le sens : vers un assèchement ou vers une humidification (Graf, 1988). Il est aussi admis qu'au-delà d'une modification globale du climat, de simples changements d'intensité, de répartition et de fréquence des précipitations sont déterminants pour la morphogenèse (Graf, 1988).

À partir de l'étude de la dynamique de la vallée du Poulvar et de la plaine de Tang-i Bulaghi à l'Holocène, trois questions principales sont abordées : l'importance de la sédimentation à l'Holocène ancien et moyen par rapport à l'Holocène récent, en relation avec l'Optimum Climatique Holocène $(\mathrm{OCH})$; l'origine de l'incision de la nappe alluviale de l'Holocène ancien et moyen ; la question des faciès sédimentaires au regard du contexte géographique. Le fait anthropique est intégré à ces réflexions dans la mesure où la région connaît une occupation qui, bien que discontinue depuis l'époque Bakoun (5 000 av. J.-C.), a pu jouer un rôle dans les transformations du milieu. Ainsi, la mise en valeur agricole et en particulier le pastoralisme, bien développé dans cette région semi-aride, aurait pu contribuer à l'érosion des sols (Kortum, 1976 ; Sumner, 1977 ; Stronach, 1978 ; Boucharlat et Benech, 2002 ; Atai et Boucharlat, 2009 ; Helwing et Seyedin, 2009).

\section{Contexte géologique, géomorpholo- gique et bioclimatique}

La vallée du Poulvar et la plaine de Tang-i Bulaghi sont situées dans la région du Fars, au centre-sud de l'Iran (fig. 1A). Ils font partie du Zagros, chaîne montagneuse orientée NW-SE qui s'étire jusqu'à la faille est-anatolienne, à l'est de la Turquie. Le Zagros résulte de la collision des plaques arabique et eurasiatique ; il est formé de plis anticlinaux orientés NW-SE, qui constituent, en Iran, un ensemble structural de chevauchement-plissement (Zagros fold-thrust belt) (Regard, 2003 ; Vernant, 2003 ; Molinaro, 2004 ; fig. 1B). Ces montagnes sont constituées avant tout de calcaires jurassiques et crétacés surmontant des grès et des roches siliceuses, eux-mêmes recouvrant d'épaisses formations évaporitiques du Cambrien. Le contexte structural commande le drainage, qui s'est organisé d'abord en fonction des plis selon une direction générale NW-SE, puis secondairement selon une direction NE-SW. La présence de sections transversales orthogonales à l'écoulement dominant est caractéristique des systèmes plissés. Ces sections témoignent ici d'un phénomène de surimposition (Fisher, 1968 ; Oberlander, 1985). En effet, la déformation récente du Plio-quaternaire, responsable du soulèvement et des plissements du Zagros, a d'abord favorisé un drainage longitudinal (Regard, 2003). Ensuite, l'érosion de la couverture peu résistante, en parallèle à la poursuite de la surrection du Zagros (Vernant, 2003), a favorisé l'apparition de cours d'eau subséquents qui ont recoupé, par surimposition, les plis inscrits dans les calcaires résistants sous-jacents datant du Crétacé. C'est à une de ces sections qu'appartient la partie de la rivière Poulvar étudiée (fig. 1C). Cette situation implique, on va le voir, une dynamique et une évolution morphologique spécifiques.

La plaine de Tang-i Bulaghi est localisée dans un synclinal constitué de roches calcaires, marnocalcaires ou encore de calcaires silicifiés datant du Crétacé (fig. 2). Cette structure est dominée au nord par un anticlinal développé dans des calcaires massifs et des calcaires dolomitiques de la fin du Jurassique. Les couches formant le synclinal sont en discordance angulaire avec les calcaires jurassiques bordant la plaine au sud, en raison de la présence d'une faille inverse orientée NW-SE, au contact montagne plaine. Un décrochement dextre orienté NE-SW délimite l'extension de la plaine à l'est. La rivière Poulvar emprunte cet accident structural dans la section étudiée ici (fig. 2). Le versant nord de la plaine a un commandement de plus de $600 \mathrm{~m}$ tandis que le versant sud culmine à un peu plus de $2000 \mathrm{~m}$, avec une dénivelée de $200 \mathrm{~m}$. Cette morphologie découpée favorise l'apport d'un volume important de matériel au fond de la plaine par l'intermédiaire des cours d'eau intermittents qui incisent les calcaires massifs, ou par les ruissellements diffus en bas de versants. Sur les piémonts se développent des cônes alluviaux (fig. 2) à la sortie de petites gorges. Vers 


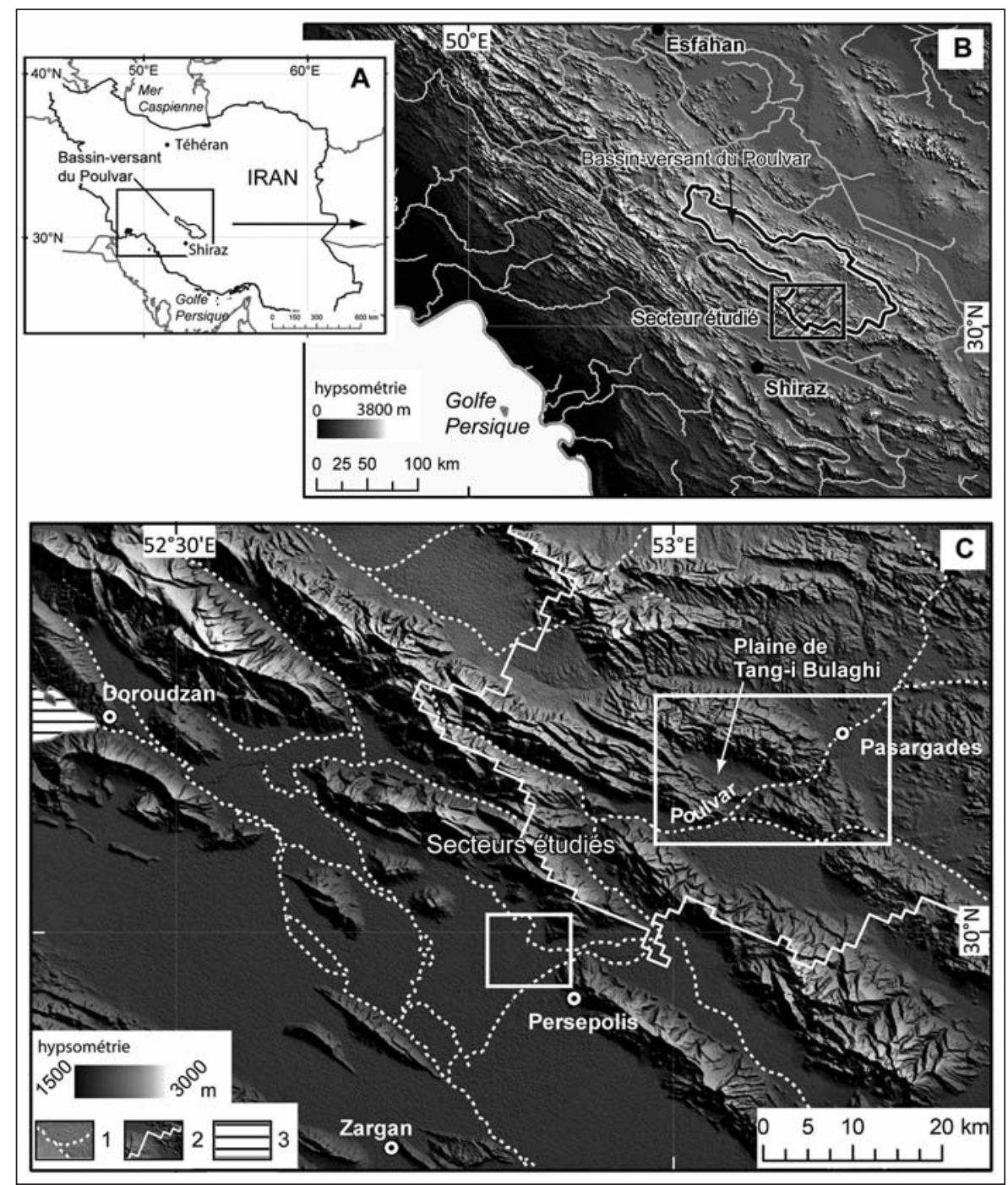

Fig. 1 - Le terrain d'étude. A : Carte de localisation. B : Le Zagros et la région d'étude. C : La région de Persépolis-Pasargades ; 1 : cours d'eau ; 2 : limites du bassin-versant du Poulvar ; 3 : lac.

Fig. 1 - Studied area. A: Location map. B: Studied area and Zagros. C: Persepolis-Pasargadae area; 1: stream; 2: Poulvar hydrographic basin; 3: lake.

Fig. 2 - Géologie et formations superficielles dans la région de PasargadesTang-i Bulaghi. 1 : écoulement pérenne 2 : écoulement temporaire ; 3 : faille ; 4 Pléistocène supérieur et Holocène (cônes alluviaux et glacis) ; 5 : Holocène (alluvions du Poulvar ; sables, limons, argiles) 6 : Holocène (alluvions et colluvions) ; 7 Holocène (épandages alluviaux grossiers) ; 8 : Pléistocène supérieur (conglomérats) ; 9 : Crétacé (marnes et calcaires argileux) ; 10 : Jurassique (calcaires, calcaires dolomitiques et dolomies) ; 11 : direction de l'écoulement du Poulvar.

Fig. 2 - Geology and surficial deposits in the Pasargadae-Tang-i Bulaghi region. 1: perennial stream; 2: temporary stream; 3: fault; 4: Late Pleistocene and Holocene (alluvial fan and pediment); 5 : Holocene (alluvial deposit from the Poulvar River; sand, silt, clay); 6: Holocene (alluvial and colluvial deposit); 7: Holocene (coarse alluvial deposit); 8: Late Pleistocene (conglomerate); 9: Cretaceous (marls and argillaceous limestone); 10 : Jurassic (limestone, dolomitic limestone and dolomite); 11: flow direction of the Poulvar river.

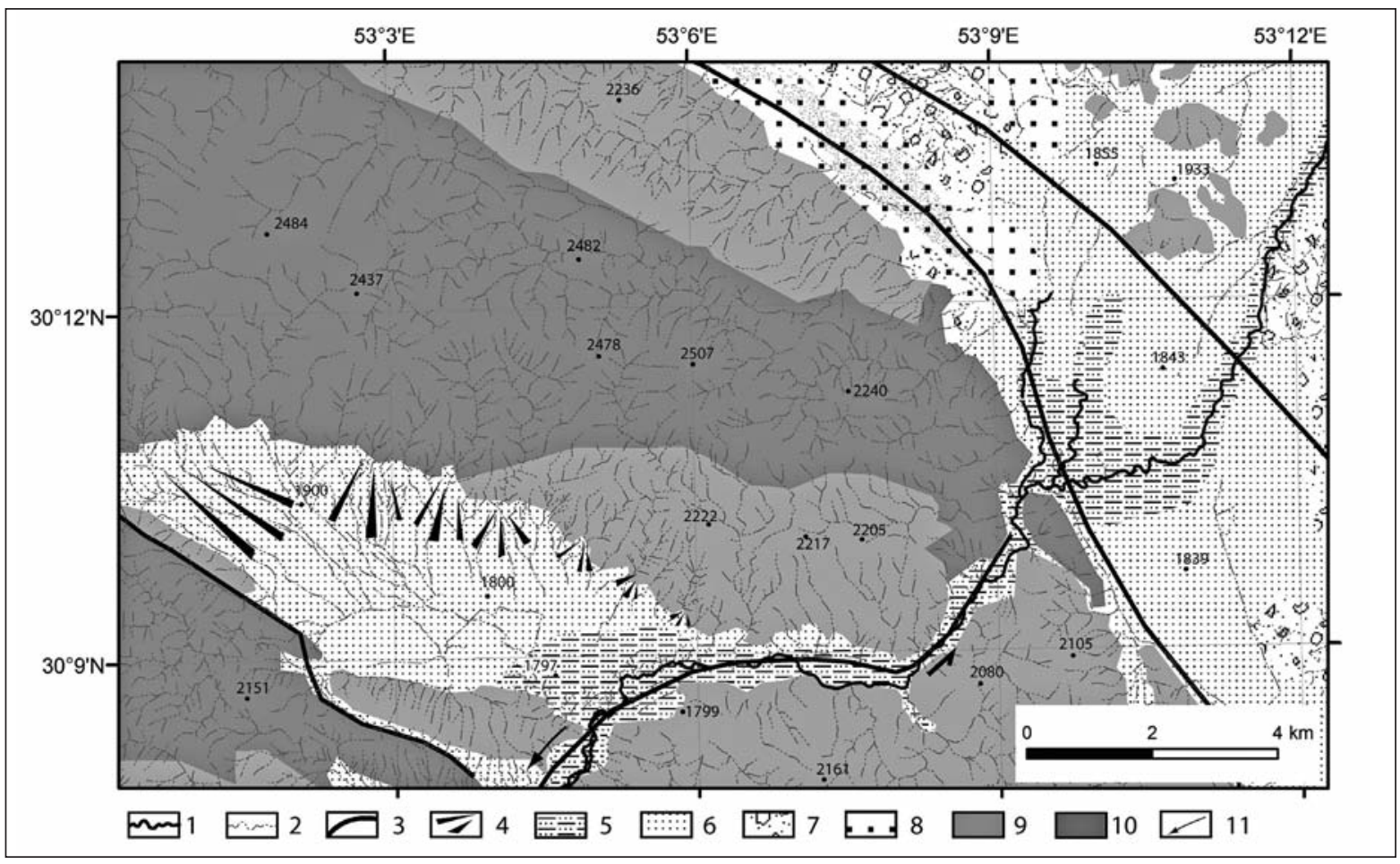


l'aval, ces cônes coalescents donnent naissance à des glacis d'épandage puis à une plaine d'épandage incisée aujourd'hui par le Poulvar. L'inclinaison de la plaine jusqu'au drain fluvial est régulière et relativement modeste sans être aussi faible que celle de certains glacis (2,5\% environ ; fig. 3$)$. La vallée du Poulvar est étroite et encaissée d'environ 200 m entre deux versants raides développés dans les calcaires (fig. 3). Le pied des versants est occupé par des cônes d'éboulis et des cônes alluviaux. La vallée est parcourue par un cours d'eau permanent caractérisé par un chenal unique dans lequel se trouvent des barres latérales alternées et de rares îles. Ce chenal est sub-rectiligne à peu sinueux (indice de sinuosité entre 1 et 1,33). Au printemps, période de hautes eaux, sa largeur est variable, mais en moyenne de 20 à $30 \mathrm{~m}$

Fig. 3 - Coupes stratigraphiques types dans les nappes alluviales, profils topographiques et emboîtement des terrasses dans la vallée du Poulvar et dans la plaine de Tang-i Bulaghi. A : les coupes stratigraphiques et leur localisation. 1 : cours d'eau ; 2 : plaine d'épandage de Tang-i-Bulaghi ; 3 : terrasses holocènes ; 4 : chenal actuel du Poulvar ; 5 : localisation des coupes stratigraphiques présentées dans l'article ; 6 : points d'observation et d'échantillonnage ; 7 : dépôt sablo-caillouteux ; 8 : dépôt limono-sableux à lentille caillouteuse ; 9 : dépôt limono-sableux ; 10 : limons ; 11 : dépôt limono-argileux ; 12 : non visible. B : Exemple d'emboîtement des terrasses holocènes dans la vallée du Poulvar.

Fig. 3 - Typical alluvial sections, topographical profiles and nested fill terraces in the Poulvar valley and in the Tang-i Bulaghi plain. A: Alluvial sections and their location. 1: stream; 2: outwash plain of Tang-i Bulaghi; 3: Holocene terraces; 4: present channel of the Poulvar River; 5: sections discussed in the text; 6: points of observation and sampling; 7: sand and gravel deposit; 8: silty-sandy deposit with coarse lenses; 9: silty-sandy deposit; 10: silt; 11: silty-argillaceous deposit; 12: not visible. B: Example of Holocene nested fill terraces in the Poulvar valley. et sa profondeur de moins d'1 m. Le rapport largeur/profondeur, assez faible, indique une forte charge en suspension. Audelà du chenal actuel, la vallée garde la trace de plusieurs anciennes nappes alluviales. La principale forme aujourd'hui une vaste terrasse dont le sommet se situe à $17 \mathrm{~m}$ du fond du chenal actuel (fig. 4). Au niveau de cette terrasse, la largeur du fond de vallée varie entre $150 \mathrm{~m}$ et $350 \mathrm{~m}$ en moyenne, avant de s'évaser progressivement à l'entrée de la plaine de Tang-i Bulaghi, sur $600 \mathrm{~m}$ de large (fig. 4). Ainsi, le Poulvar, aujourd'hui limité à un chenal étroit, a une histoire complexe, illustrée par des phases d'incision et d'aggradation durant lesquelles la totalité du fond de vallée est occupée par la rivière.
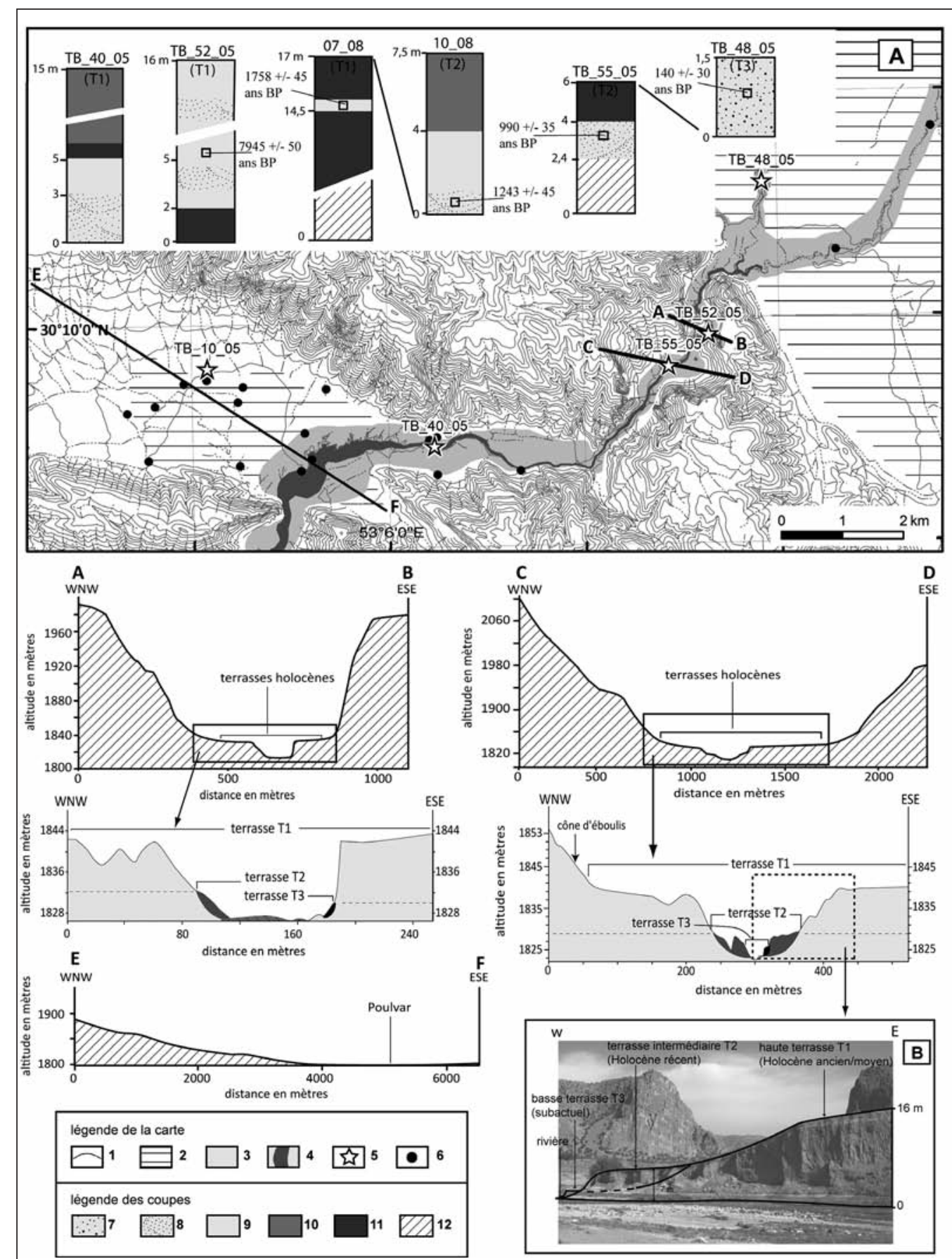


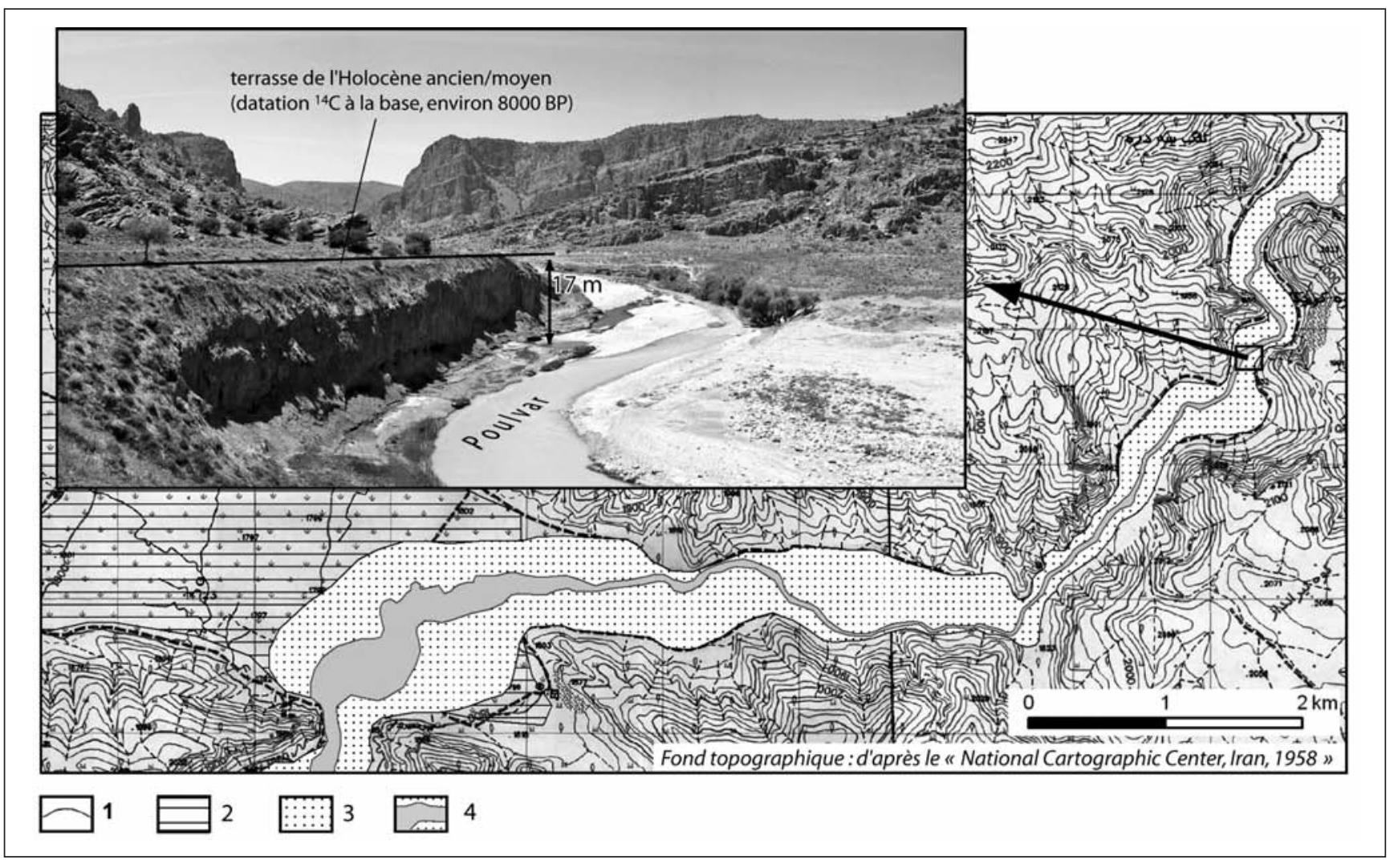

Fig. 4 - La terrasse de l'Holocène ancien dans la vallée du Poulvar. 1 : courbes de niveau (équidistance $20 \mathrm{~m}$ ) ; 2 : plaine d'épandage de Tang-i-Bulaghi ; 3 : terrasses holocènes ; 4 : chenal actuel du Poulvar.

Fig. 4 - Early Holocene terrace in the Poulvar valley. 1: contour line (20 m interval); 2: outwash plain of Tang-i Bulaghi; 3: Holocene terraces; 4: present channel of the Poulvar River.

La région de Pasargades est située autour de $30^{\circ} 10^{\prime}$ de latitude nord et $53^{\circ} 10^{\prime}$ de longitude ouest, à une altitude d'environ $1800 \mathrm{~m}$. Les données climatiques proviennent des stations météorologiques de Doroudzan et de Zargan situées, pour la première, à environ $75 \mathrm{~km}$ à l'ouest, sur la même latitude et pour la seconde à $65 \mathrm{~km}$ au sud-ouest (fig. 1C). Elles portent sur des relevés réalisés entre 1988 et 2003, et sont fournies par 1'I.R. of Iran Meteorological Organisation (IRIMO, http://www.irimo.ir). Selon ces statistiques, la région reçoit entre $350 \mathrm{~mm}$ et $500 \mathrm{~mm}$ de précipitations annuelles moyennes. La température moyenne annuelle sur la période est d'environ $18^{\circ} \mathrm{C}$. Les diagrammes ombrothermiques de ces stations sont typiques d'un climat semiaride de type méditerranéen (fig. 5A et B). En effet, on note deux saisons bien distinctes : un hiver assez rigoureux, avec des températures moyennes de $6,5-7^{\circ} \mathrm{C}$ entre décembre et février, durant lequel tombent 57-63\% des précipitations (respectivement pour Zargan et Doroudzan) ; un été chaud et sec, avec une moyenne des températures de juin à septembre de $28-29^{\circ} \mathrm{C}$. La sécheresse s'étire de mai à octobre (fig. 5A et $\mathrm{B})$, avec des précipitations très faibles voire inexistantes $(0,4 \%$ des précipitawww.irimo.ir). tions tombent entre juin et septembre). Le gel est présent, surtout au mois de janvier, dont la moyenne des températures minimum est négative à Zargan $\left(-1,5^{\circ} \mathrm{C}\right)$ et proche de zéro à Doroudzan $\left(0,5^{\circ} \mathrm{C}\right)$. Le nombre moyen de jours durant lesquels les températures minimales sont inférieures ou égales à $0^{\circ} \mathrm{C}$ est respectivement de 22 à Zargan et de 15 à Doroudzan. Deux éléments influencent fortement le climat de la région : la latitude, identique à celle du Caire, qui ex-

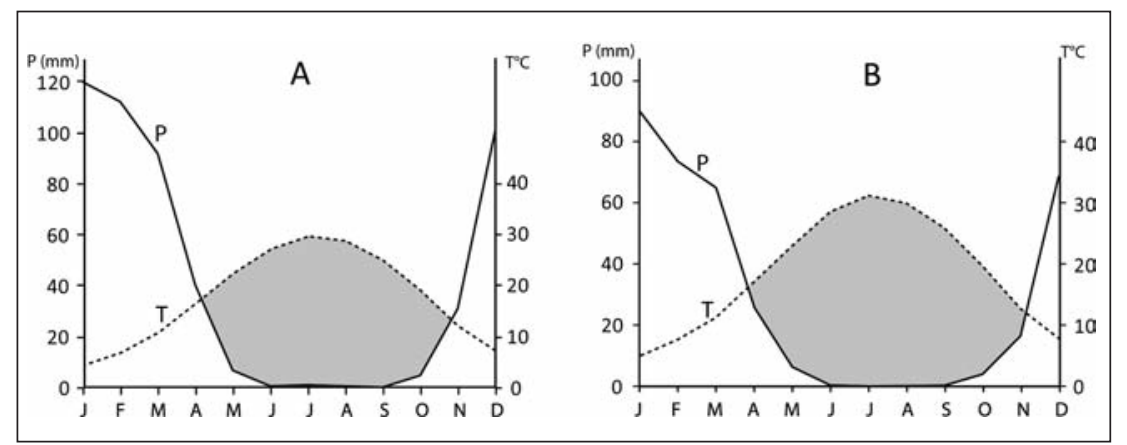

Fig. 5 - Données climatiques dans la région de Pasargades-Persépolis (1988-2003). A : Diagramme ombrothermique de la station de Doroudzan. B : diagramme ombrothermique de la station de Zarqan. D'après l'organisation météorologique de la république islamique d'Iran (IRIMO, http://www.irimo.ir).

Fig. 5 - Climatic data for the Pasargadae-Persepolis region (1988-2003). A: Ombrothermic diagram of the Doroudzan station. B: Ombrothermic diagram of the Zarqan station. From the Islamic Republic of Iran Meteorological Organisation (IRIMO, http:// 
plique une certaine douceur du climat et des précipitations peu abondantes, et l'altitude, qui explique la relative fraicheur des températures en hiver, le gel fréquent et une moyenne annuelle inférieure à ce qui s'observe généralement en domaine méditerranéen. Un tel contexte climatique favorise la morphogenèse, marquée par une vive érosion en raison des pentes fortes, des mécanismes de désagrégation mécanique des roches tels que la cryoclastie et la concentration des précipitations sur seulement 50 jours en moyenne par an. La végétation appartient au domaine kurdo-iranien. Elle se caractérise par une steppe arborée à genévrier, pistachier et amandier. On trouve également Artemisia et Quercus Brantii (Zohary, 1973 ; Frey et Kurschner, 1989). Les espèces sont peu nombreuses et la végétation est lâche. L'agriculture actuelle est caractérisée par la culture des céréales et des légumineuses. Elle est dominée de manière écrasante par l'irrigation par pompage ou par dérivation dans le fond des plaines, tandis que les versants sont rarement exploités, hormis pour l'arboriculture et pour les pâtures.

\section{Méthodes d'étude}

L'analyse de documents cartographiques, iconographiques et climatologiques a permis de poser le cadre structural et climatique régional et d'avoir une première idée du contexte géomorphologique dans la vallée et dans la plaine de Tangi Bulaghi. Cette étape préliminaire a été suivie par une étude de terrain centrée sur le remplissage sédimentaire et l'évolution morphologique, par l'analyse de coupes stratigraphiques naturelles et de la topographie de détail. Des coupes stratigraphiques naturelles ont été étudiées dans le chenal actuel de la vallée du Poulvar. Dans la plaine de Tang-i Bulaghi, de nombreuses coupes stratigraphiques artificielles, due à des travaux d'édification d'un barrage, ont permis d'avoir un aperçu de la couverture sédimentaire. Au total, 15 coupes stratigraphiques ont été étudiées dans la vallée et dans la plaine. Les dépôts étant remarquablement homogènes, seules quelques coupes stratigraphiques représentatives ont été présentées ici. L'étude du profil transversal de la vallée et du chenal actuel n'a pas donné lieu à des mesures topographiques systématiques mais deux profils détaillés du

Tab. 1 - Datations radiocarbone.

Tab. 1 - Radiocarbon dates. fond de vallée ont été réalisés à l'aide d'une station totale Leica, qui montrent distinctement les différents niveaux de sédimentation encore en place. Enfin, un certain nombre d'analyses en laboratoire ont permis d'affiner l'étude sédimentaire et chronologique. Une première série d'analyses granulométriques a été menée sur 11 échantillons pris dans les différentes nappes alluviales de la vallée du Poulvar. Il s'agissait d'avoir un premier aperçu de la texture au sein des différentes séquences en se fondant sur l'analyse de la fraction fine $(<0,63 \mathrm{~mm})$ et de la fraction grossière $(>0,63 \mathrm{~mm})$. Les analyses ont été réalisées par V. Gaertner au Laboratoire rhodanien de géographie de l'environnement de l'université Lyon 2 à l'aide d'un granulomètre laser (Master sizer 2000 de chez Malvern). Des analyses calcimétriques sur chaque échantillon ont été réalisées en parallèle. Des mesures plus systématiques devraient être menées sur des échantillons prélevés lors d'une nouvelle mission qui s'est déroulée en 2008. Ces analyses ont été complétées par des datations ${ }^{14} \mathrm{C}$ réalisées au Centre de datation par le radiocarbone de Lyon et au Centro di Datazione e Diagnostica de l'université de Salente (Italie). Cinq échantillons ont donné des résultats satisfaisants (tab. 1).

\section{Dynamique sédimentaire de la rivière Poulvar et de la plaine de Tang-i Bulaghi}

\section{Terrasses, formes de piémont et chronologie}

Les dépôts de la vallée du Poulvar se caractérisent très majoritairement par des nappes alluviales limono-sableuses de forte épaisseur. Ces nappes ont été incisées par le cours d'eau et dessinent aujourd'hui des terrasses dominant le fond de vallée. On note trois terrasses alluviales emboîtées numérotées T1 à $\mathrm{T} 3$ de la plus ancienne à la plus récente, bien mises en évidence par les profils topographiques (fig. 3). La terrasse la plus ancienne domine les bancs latéraux du chenal actuel d'environ $17 \mathrm{~m}$. Une date de $7945 \pm 550$ BP (Ly-3364) obtenue sur un charbon dans un niveau situé à environ $5 \mathrm{~m}$ de la base de la terrasse apporte une première indication chronologique. Cette terrasse constitue l'unité morphologique principale du paysage. Elle occupe l'essentiel du fond de la

\begin{tabular}{|c|c|c|c|c|c|c|}
\hline Site (voir fig. 1) & $\begin{array}{l}\text { Numéro et code } \\
\text { du laboratoire }\end{array}$ & $\begin{array}{l}\text { Profondeur } \\
\text { (en } \mathrm{m})\end{array}$ & Matériel & $\begin{array}{l}\text { Date }{ }^{14} \mathrm{C} \text { BP } \\
\quad(1 \sigma)\end{array}$ & $\begin{array}{l}\text { D 13C } \\
\text { (en \%o) }\end{array}$ & Age calibré $(2 \sigma)^{\star}$ \\
\hline Tang-i Bulaghi & Ly-3364 & 12 & charbon de bois & $7945 \pm 50$ & non transmis & 7 055-6 652 av. J.-C. \\
\hline Tang-i Bulaghi & Ly-3365 & 2 & charbon de bois & $990 \pm 35$ & non transmis & 995-1 156 ap. J.-C. \\
\hline Pasargades & Ly-3363 & 0,8 & charbon de bois & $140 \pm 30$ & non transmis & post 1650 ap. J.-C. \\
\hline Persépolis & LTL4127A & 4,5 & charbon de bois & $1243 \pm 45$ & non transmis & 680-820 ap. J.-C. \\
\hline Persépolis & LTL4130A & 2 & charbon de bois & $1758 \pm 45$ & non transmis & 220-350 ap. J.-C. \\
\hline
\end{tabular}

* Calibration établie selon M. Stuiver et al. (1998) 
vallée (fig. 3) et se rattache, latéralement, aux dépôts tapissant la plaine de Tang-i Bulaghi. Le second niveau délimite une terrasse emboîtée à $10 \mathrm{~m}$ en contrebas du sommet de la nappe alluviale précédente. Cette terrasse possède une hauteur d'environ $6 \mathrm{~m}$. Un charbon situé dans un niveau cendreux à environ $4 \mathrm{~m}$ de la base de la terrasse a été daté de $990 \pm 35$ BP (Ly-3365), soit entre 995 et 1156 ap. J.-C. L'étendue latérale de cette terrasse est assez faible en comparaison à la précédente. Elle domine les deux bords du chenal actuel sur quelques dizaines de mètres de large (fig. 3). Enfin, une très basse terrasse est emboîtée dans la précédente à environ $3 \mathrm{~m}$ en contrebas (fig. 3). Son épaisseur visible est de 2-3 m. Une datation sur charbon de bois donne la moitié supérieure de cette petite nappe alluviale à $140 \pm 30 \mathrm{BP}$ (Ly-3363). Des nappes alluviales ont été observées et étudiées dans le chenal du Poulvar, au sein de la plaine de Marv Dacht (Persepolis), plus en aval (fig. 1C et fig. 3). On observe la même succession de trois niveaux de dépôt alluviaux. Les épaisseurs sont sensiblement les mêmes : 16$18 \mathrm{~m}$ pour le niveau supérieur (T1), 6-8 m pour le niveau intermédiaire (T2) et environ $2 \mathrm{~m}$ pour le bas niveau (T3). Des échantillons de charbon ont été datés à la base du niveau intermédiaire T2 (fig. 3, coupe 10_08) et dans la partie sommitale du niveau supérieur T1 (fig. 3, coupe 04_08). Les dates obtenues sont respectivement $1243 \pm 45$ BP (LTL4127A) et $1758 \pm 45 \mathrm{BP}$ (LTL4130A). Si la première date corrobore la date obtenue plus en amont, la seconde soulève des interrogations qui seront discutées plus tard. La plaine de Tang-i Bulaghi est caractérisée par la présence de cônes alluviaux évoluant en glacis vers l'aval. Il n'y a pas d'étagement des formes, ce qui rend difficile la mise en évidence d'une chronologie du comblement de la plaine. Vers l'aval, les dépôts des glacis passent sans rupture de pente aux dépôts de la terrasse supérieure (T1) de la plaine alluviale du Poulvar (fig. 3). Sur les cônes-glacis se trouvent des sites archéologiques dont certains ont fait l'objet de fouilles. Il s'agit de sites Achéménide (550-350 av. J.-C.), Sassanide (700-200 av. J.-C.) et Bakoun (5 000 av. J.-C.) en surface ou en subsurface (1 à $2 \mathrm{~m}$; fig. 6). Ces sites constituent les seuls éléments de chronologie que nous ayons et donnent un terminus ante quem pour les formations de la plaine.

L'étude d'une quinzaine coupes stratigraphiques dans les nappes alluviales a permis de dresser un premier aperçu des processus morphogénétiques dans la vallée et dans la plaine (les coupes stratigraphiques sont localisées sur la fig. 3A).

- La plupart des coupes stratigraphiques observées dans la nappe alluviale supérieure (T1) montrent des niveaux à dominante limoneuse (limono-argileux et limono-sableux). Le faciès est en général très homogène, de structure massive, dans laquelle la stratification est souvent peu visible. Ces niveaux fins sont entrecoupés par de rares lentilles sablocaillouteuses formées de galets bien roulés, dont le grand axe atteint $10 \mathrm{~cm}$. Une coupe stratigraphique observée à l'amont de la vallée de Poulvar (point 52-05, fig. 3) montre un niveau limono-argileux à la base, sur les deux premiers mètres au-dessus du bord du chenal actuel [sables $(\mathrm{S})=$ $9 \%$; limons $(\mathrm{L})=69 \%$; Argiles $(\mathrm{A})=22 \%$ )]. La stratification est peu marquée, en dehors d'une unité sableuse de
$10 \mathrm{~cm}$ d'épaisseur, qui marque le sommet du niveau. Des lentilles de cailloutis s'observent parfois. Au-dessus, les sédiments sont toujours très limoneux, mais avec une plus grande proportion de sables $(\mathrm{S}=18 \% ; \mathrm{L}=68 \% ; \mathrm{A}=$ $14 \%$ ). Ce faciès semble se prolonger jusqu'au sommet de la coupe stratigraphique, mais en raison de sa hauteur, nous n'avons pas pu faire d'observation précise. On note, là aussi, la présence de lentilles sablo-caillouteuses grossières mal classées, caractérisées par des cailloux bien roulés dont le grand axe peut dépasser $10 \mathrm{~cm}$. Des séries alternant de fines couches limono-sableuses et argileuses de quelques centimètres s'observent parfois dans ce niveau. Ces sédiments sont moyennement carbonatés : la calcimétrie indique des taux de carbonates compris entre $34 \%$ et $36 \%$. Une autre coupe stratigraphique, située à quelques centaines de mètres en aval, montre des dépôts limono-sableux sur $1 \mathrm{~m}$ (au-dessus des 3,5 premiers mètres de la coupe non visibles), puis
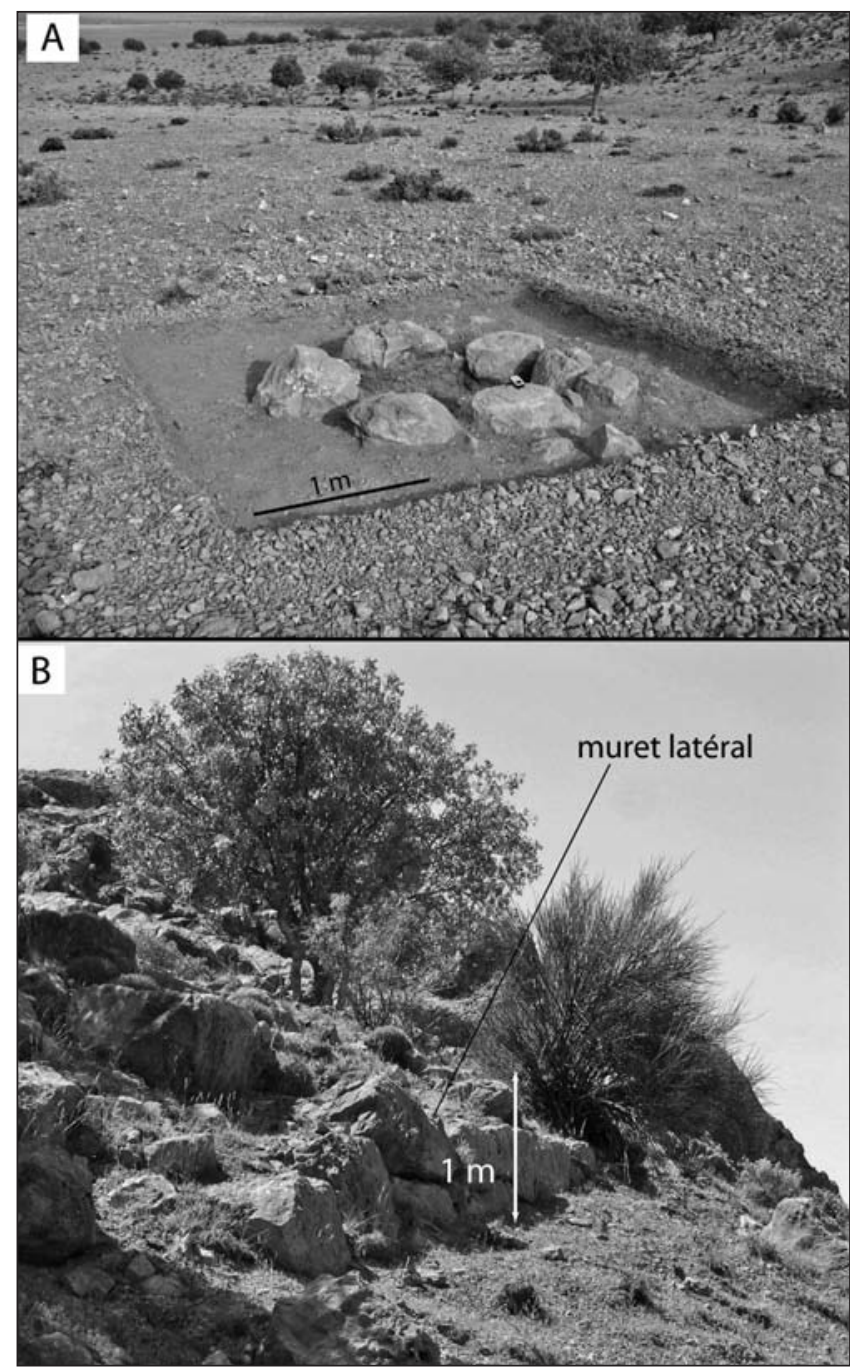

Fig. 6 - Vestiges archéologiques dans la région de Tang-i Bulaghi. A : tombe Sassanide sur la partie médiane d'un cône alluvial. $B$ : vestiges d'un canal Achéménide sur le versant est de la vallée du Poulvar.

Fig. 6 - Archaeological remains in the Tang-i Bulaghi region. A: Sassanid tomb on the median part of an alluvial fan. B: remains of an Achaemenid canal on the east slope of the Poulvar valley. 
un niveau limoneux, très homogène, massif, jaunâtre, légèrement oxydé, sur au moins $3 \mathrm{~m}$. Une stratification très discrète se devine, marquée notamment par un niveau à charbon de bois à 2,2 $\mathrm{m}$ de la base. Ce dépôt est surmonté ou recoupé par plusieurs lentilles de cailloutis dont une de $2 \mathrm{~m}$ d'épaisseur. Au-dessus, les niveaux restent très limoneux et argileux. Plus à l'aval encore, à l'entrée de la plaine de Tang-i Bulaghi, la disposition stratigraphique est un peu différente. Ici, une coupe stratigraphique (fig. 3, point 40-05) est constituée, à la base, d'un niveau sablo-caillouteux sur au moins $3 \mathrm{~m}$. Les cailloux sont peu émoussés et peu arrondis. Au-dessus se trouve un dépôt homogène formé de limons et de sables sur environ $2 \mathrm{~m}$, se terminant par une passée caillouteuse de $20 \mathrm{~cm}$. Un dépôt fin à structure massive et compacte recouvre le dépôt précédent et semble se poursuivre jusqu'au sommet de la coupe stratigraphique, sur 8$10 \mathrm{~m}$. Un échantillon de sédiments pris à la base de ce niveau très homogène montre une texture argilo-limoneuse sans sable $(\mathrm{L}=50 \% ; \mathrm{A}=50 \%)$. Ici aussi, le taux de carbonates est moyen $(31,7 \%)$.

- La nappe alluviale suivante (T2), d'environ 6 m d'épaisseur, se caractérise généralement par la présence d'un épais niveau sablo-caillouteux. Ce niveau surmonte un dépôt limono-sableux et parfois un dépôt limoneux très compact appartenant probablement à la nappe alluviale antérieure (T1), tandis qu'il est coiffé d'un dépôt fin limono-sableux. Une coupe stratigraphique (fig. 3, point 55-05) montre, à la base, un dépôt limono-sableux $(\mathrm{S}=26 \%$; $\mathrm{L}=$ $60 \% ; \mathrm{A}=14 \%$ ), sur $1,7 \mathrm{~m}$ (les 2,4 premiers mètres ne sont pas visibles); ce niveau passe latéralement à une accumulation caillouteuse grossière ; au-dessus s'observe un niveau argileux réducteur gris-verdâtre sur $40 \mathrm{~cm}$, surmontée d'une passée cendreuse de quelques centimètres ; ces niveaux sont recouverts par une accumulation caillouteuse hétérométrique (galets jusqu'à $15 \mathrm{~cm}$ de grand axe) sur 40 à $50 \mathrm{~cm}$, puis par un épais dépôt limono-argileux légèrement pédogénéisé sur $1,9 \mathrm{~m}(\mathrm{~S}=14 \% ; \mathrm{L}=67 \% ; \mathrm{A}=19 \%)$, avec passées caillouteuses. Dans le cas de ce dépôt, la carbonatation est plus importante. La calcimétrie indique des taux de carbonates compris entre $46 \%$ et $48 \%$.

- La nappe alluviale suivante (T3) est souvent grossière et épaisse de 2-3 m d'épaisseur. Le dépôt est sablo-caillouteux, constitué de galets bien roulés. Une coupe stratigraphique (fig. 3, point 48-05) montre, dans sa partie supérieure visible sur $1,5 \mathrm{~m}$, une alternance de couches sablo-caillouteuses $(30-50 \mathrm{~cm})$ et de couches sableuses $(5-20 \mathrm{~cm})$. Mais d'autres coupes stratigraphiques montrent que cette terrasse possède une grande variété de faciès. Ainsi, on observe des dépôts plus fins, limono-sableux, contenant des niveaux caillouteux. Cette nappe alluviale a été incisée de 3-4 m et se réduit souvent à une simple banquette en bordure de chenal.
L'emboîtement des différentes formations est bien visible dans certaines portions du chenal actuel (fig. 3B et fig. 7). Enfin, dans le fond du chenal s'observent des dépôts grossiers subactuels : bancs de chenal et barres latérales, parfois recouverts vers l'aval de dépôts limoneux.

- Dans la plaine de Tang-i Bulaghi, plusieurs coupes stratigraphiques ont pu être étudiées dans la partie médiane des cônes, au contact avec le glacis. Le matériel est hétérométrique, montrant parfois des niveaux fins. Une coupe stratigraphique relevée dans la partie nord de la plaine (fig. 3, point 10_05) montre une succession d'unités sédimentaires plus ou moins grossières constituées de sables, cailloutis et blocs dans une matrice limoneuse, entre lesquelles s'observent des couches limoneuses ou limono-argileuses d'épaisseur variable. Les niveaux hétérométriques montrent une légère recristallisation des carbonates, plus forte à la base de la coupe, en raison du battement de la nappe phréatique. Cette coupe stratigraphique est typique des épandages sur les cônes-glacis des piémonts en milieu semi-aride. Les cailloutis et cailloux sont anguleux, souvent plats, allongés dans le sens de la pente et traduisent la faible compétence des écoulements de surface. Certaines unités sédimentaires plus grossières résultent du dépôt de matériel par les chenaux divagants à la surface du cône-gla-

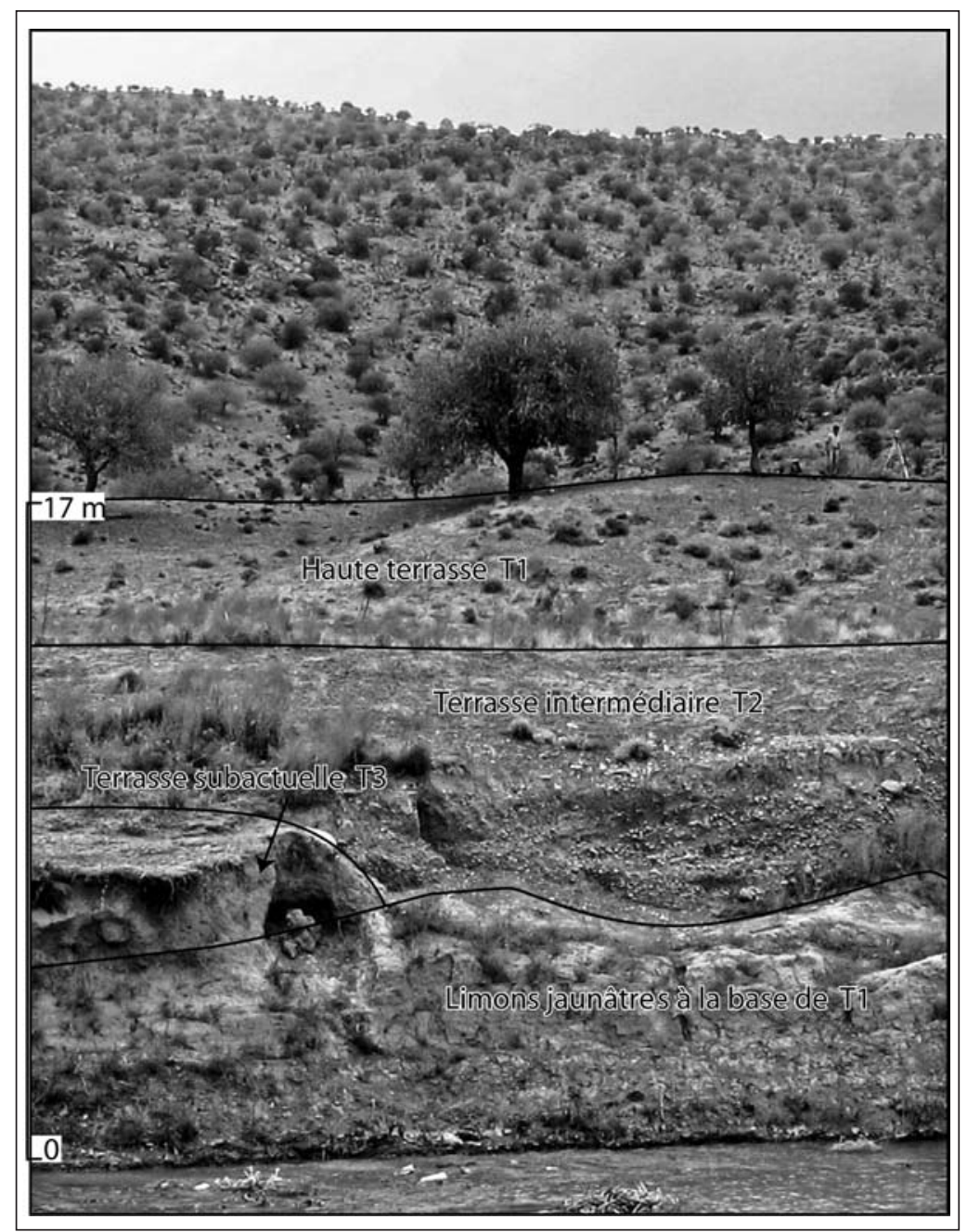

Fig 7 - Emboîtement des terrasses dans la vallée du Poulvar.

Fig. 7 - Cut-and-filled terraces in the Poulvar valley. 
cis. Enfin, les couches limoneuses témoignent soit d'une simple variation de faciès due au déplacement d'un chenal, soit d'un ralentissement de la morphogenèse, voire d'une phase de stabilité morphologique. En général, la partie supérieure des dépôts, au contact entre les cônes et les glacis d'épandage est constituée d'un matériel à dominante limoneuse entrecoupé d'unités sédimentaires caillouteuses plus ou moins épaisses. Plus à l'aval, dans la plaine d'épandage, ce sont les limons qui dominent. La présence de sites Bakoun en subsurface indique que ces cônes-glacis datent très probablement du Dernier Maximum Glaciaire (DMG) et du début de l'Holocène. L'absence de datation empêche pour l'instant de replacer plus précisément cette coupe stratigraphique dans le temps.

\section{Interprétation des formes et des formations}

Dans la haute terrasse (T1), certaines coupes stratigraphiques montrent, à leur base, des niveaux de cailloutis et de galets peu émoussés et peu arrondis. Le faible émoussé de ce matériel et sa nature (galets et cailloutis de calcaire) indiquent une origine proche, probablement issue des versants immédiats de la vallée façonnés dans des calcaires jurassiques et crétacés. D'après nos observations, ces dépôts semblent appartenir à des cônes alluviaux développés en bas de versant puis partiellement remaniés et recouverts par les alluvions du Poulvar. Il n'y aurait donc pas de synchronisme entre les dépôts grossiers de versant et les dépôts fins du cours d'eau. D'autres coupes stratigraphiques ne montrent pas ces niveaux basaux grossiers car le chenal incise la nappe alluviale loin des versants. En revanche, on observe assez régulièrement des lentilles de matériel grossier bien émoussé. Dans ce cas, il s'agit de paléochenaux qui témoignent que le cours d'eau a divagué dans des alluvions à dominante fine. Il y a donc une différence claire entre les restes d'alluvions grossières en place en pied de versant et les lentilles de matériel remanié dans le chenal du Poulvar. Ces observations nous conduisent à supposer, avec toute la prudence nécessaire car nous n'avons pour l'instant que deux dates pour la haute terrasse $(7945 \pm 50 \mathrm{BP}$ dans la moitié inférieure et $1758 \pm 45$ BP dans la partie sommitale), que la mise en place des cônes alluviaux a eu lieu lors d'une phase d'érosion intense qui pourrait être associée au DMG. En plus de ces dates, nous savons que des sites de l'époque Bakoun (5 000 av. J.C.) se situent en subsurface des cônes alluviaux, que ce soit sur les bas de versants de la vallée du Poulvar ou dans la plaine de Tang-i Bulaghi. Il est donc assez clair qu'ils se sont édifiés, pour la plupart d'entre eux, avant l'Holocène ancien. Durant cette phase morphologiquement active, le chenal du Poulvar s'est probablement remblayé de sédiments grossiers, dont on observe aujourd'hui des témoignages remobilisés dans des paléochenaux de la haute terrasse (T1), de la terrasse intermédiaire (T2), et dans les barres de chenal contemporaines. La phase d'intense érosion hydrique du DMG a également été observée plus au sud, dans la plaine de Persépolis, dans laquelle débouche le Poulvar. D'après une étude de M. Kehl et al. (2005), cet événement se manifeste par une accumulation d'environ $10 \mathrm{~m}$ de sédiments.
Les dépôts alluviaux fins (T1) qui recouvrent la base des cônes et la nappe alluviale du DMG se sont déposés très vraisemblablement durant l'Holocène ancien et moyen. Encore une fois, les arguments chronologiques sont peu nombreux mais la date obtenue dans la partie basale de cette terrasse (7 $945 \pm 50 \mathrm{BP}$ ) et les observations géomorphologiques vont clairement dans ce sens. Nous avons observé cette phase de sédimentation dans la plaine de Persépolis. Elle se caractérise par une nappe alluviale qui s'observe dans toute la plaine. Les sédiments sont très similaires à ceux observés en amont ; ils sont essentiellement limoneux et remarquablement homogènes, s'apparentant à des lœss ou des pseudo-lœss, dans la mesure ou ils ont été remaniés. On y observe ponctuellement des chenaux constitués d'alluvions grossières (graviers et sables). La date obtenue (1 $758 \pm 45 \mathrm{BP})$ sur des charbons dans le niveau sommital de la terrasse, à l'entrée de la plaine de Persépolis, est très récente. Elle ne semble pas pouvoir se conjuguer avec la date de la nappe alluviale suivante ( $c f$. infra). Cet épisode de sédimentation est aussi mis en évidence par M. Kehl et al. (2005) dans l'étude morphopédologique de la plaine de Persépolis. Les dépôts décrits sont limoneux et forment également la majeure partie des débris qui tapissent la plaine. Ils sont également assimilés à des lœss ou des pseudo-lœss (Kehl et al., 2005). La différence entre les dépôts de la vallée du Poulvar et ceux de la plaine de Persépolis porte sur les taux de carbonates, plus faible pour les premiers (30-40\% contre plus de $50 \%$; Kehl et al., 2005). Il semble donc qu'une phase majeure de dépôt lœssique soit intervenue durant une période beaucoup plus froide, probablement à la fin du dernier Glaciaire. Ces lœss ont tapissé le fond des plaines et recouvert les versants. Ils ont été ensuite érodés, transportés puis déposés entre la fin du DMG et le début de l'Holocène. L'absence actuelle de sol sur les versants, sauf en de rares endroits, l'épaisseur de la nappe alluviale, son faciès et la présence de site Bakoun quasiment en surface sur les cônes alluviaux, semblent confirmer ce scénario.

Après l'incision de la nappe alluviale supérieure T1 sur une quinzaine de mètres, une nappe alluviale (T2) de 4 à 6 m d'épaisseur aujourd'hui, s'est mise en place. D'après les deux dates obtenues sur cette nappe alluviale, sa mise en place s'amorce un peu avant $1250 \mathrm{BP}$ et se poursuit pendant quelques centaines d'années. Vers 990 BP, les deux tiers de cette nappe sont déjà en place. La nappe possède un niveau grossier sablo-caillouteux récurrent à la base, qui signale une forte énergie de transport et le retour d'une phase d'érosion dans la région. Le style fluvial des cours d'eau est probablement en tresses, à l'image de ce qui s'observe fréquemment en milieu montagnard (Bravard et Petit, 1997). Il est possible également qu'il s'agisse d'un cours d'eau assez proche de l'actuel, relativement peu sinueux et à bancs latéraux. Le niveau supérieur est constitué d'un matériel fin, tantôt limono-sableux, tantôt limono-argileux. Ce matériel a pour origine l'érosion de la nappe alluviale antérieure. Il signale une fin de phase durant laquelle le cours d'eau possède une énergie de transport moindre. En parallèle, le faciès du dépôt montre qu'il a subi une pédogenèse. L'achèvement de la mise en place de la nappe alluviale T2 semble donc se 
faire dans des conditions de morphogenèse suffisamment stables pour être propices au développement d'un un sol. La nappe alluviale T2 est ensuite incisée sur environ $6 \mathrm{~m}$ et une nappe alluviale (T3) de 2 à $3 \mathrm{~m}$ d'épaisseur se met en place. Lorsque cette nappe est décapée et au vu du faciès des niveaux que l'on observe parfois à la base de la terrasse intermédiaire T2, on peut supposer que l'incision précédente a creusé également dans la nappe antérieure T1 (fig. 7). Cette ultime nappe alluviale se trouve parfois dans le prolongement de la précédente, elle-même partiellement décapée. Elle forme aujourd'hui une terrasse étroite s'apparentant à une banquette peu marquée en bordure du chenal. Le dépôt signale un retour de l'alluvionnement relativement grossier durant lequel le chenal est sans doute à l'image de l'actuel, peu sinueux, avec une forte charge solide et encombré de bancs latéraux sablo-caillouteux. La nappe est ensuite très largement décapée par le cours d'eau à la suite de son incision et par le sapement latéral actuel. Il semble, là aussi, que l'incision continue de creuser dans les dépôts de la nappe $\mathrm{T} 1$.

\section{Discussion}

Durant l'Holocène ancien et moyen, la vallée du Poulvar a été marquée par un bilan de dissection négatif. Cette situation résulte d'importants apports latéraux dus à l'érosion de la couverture pédologique sur les versants. La «prédominance des mécanismes de façonnement des interfluves sur les mécanismes de façonnement des lits fluviaux » (Tricart, 1977 , p. 189) a ici plusieurs origines, mais ce qui semble dominer est un événement climatique de grande ampleur, l'OCH. Cet événement, situé entre 9000 BP et 6000 BP environ, est caractérisé dans les régions méditerranéennes et proche-orientales par un climat plus chaud et plus humide en raison de la remontée de la zone de convergence intertropicale (ZCIT ; Roberts, 1989 ; Sanlaville, 1997 ; Blanchet et al., 1998 ; Rossignol-Strick, 1999). Les études paléoenvironnementales et paléoclimatiques portant sur la périphérie du Golfe Persique confirment son existence dans la région. Rappelons que la région du Golfe et en particulier l'Iran est caractérisée, à la fin du Pléistocène supérieur, par un climat froid et semi-aride (Bobek, 1963 ; Van Zeist et Bottema, 1977 ; Ganji, 1978). La végétation dominante est une steppe à armoise (Van Zeist et Bottema, 1991). D'après V. Regard et al. (2006), qui ont daté au Béryllium 10 l'arrêt de l'alluvionnement sur un cône alluvial au sud-est de l'Iran, l'aridité s'accroît durant le DMG, entre 20000 BP et 17000 BP. Après le retour d'un climat plus arrosé, le Dryas récent se manifeste par une phase sèche débutant vers $12800 \pm 1000$ BP. Puis, vers $9500-9000 \mathrm{BP}$, le climat se modifie fortement vers une humidification. Le faciès des dépôts sédimentaires dans le Golfe Persique change à cette date, passant de boues aragonitiques à des marnes. Cela est interprété comme la transition entre un climat semi-aride et un climat plus humide (Uchupi et al., 1999). Ces dépôts qui atteignent $20 \mathrm{~m}$ d'épaisseur proviennent en grande partie du Zagros et sont dus à la forte déglaciation entre $9500 \mathrm{BP}$ et $8500 \mathrm{BP}$ (Uchupi et al., 1999). L'existence d'un OCH entre 9000 BP et 6000 BP est égale- ment mise en évidence dans le bassin de Persépolis par la présence de paléosols dans des lœss qui témoignent d'un accroissement des précipitations sur la période (Kehl et al., 2005). Cependant, dans un article plus récent, ces même auteurs émettent l'hypothèse de la persistance, dans cette région, de conditions climatiques plus froides et plus sèches jusqu'à 7000 BP (Kehl et al., 2009). A partir de 6000 BP, le climat actuel, plus aride, se met en place. V. Regard et al. (2006) observent un changement net vers une aridification vers $5600 \pm$ 600 BP. C'est d'ailleurs à partir de cette date que s'amorce le passage de la savane au désert, en particulier en Arabie, au Sahara ou encore dans le Thar (Street-Perrot et al., 1985). Enfin, vers 4500 BP s'établit le régime de pluies d'hiver tel qu'on le connaît aujourd'hui (Stevens et al., 2001).

Cette chronologie, qui reste à préciser pour la vallée du Poulvar, pose la question du rôle de l'OCH en milieu semiaride. En effet, théoriquement ce type d'événement ne favorise pas la sédimentation mais encourage plutôt l'incision des chenaux en raison d'une stabilisation des versants par la végétation. D'ailleurs, pour M. Kehl et al. (2009), l'épaisse nappe alluviale T1 se serait déposée dans des conditions plus sèches et plus froides. Cependant, comme nous l'avons vu plus haut, le faciès des dépôts n'est pas caractéristique d'un climat sec. Ces dépôts témoigneraient $a$ contrario d'un retour à des conditions plus humides autour de 8 000-9 000 BP. Comment alors expliquer cette sédimentation et sa nature dans la rivière Poulvar? L'hypothèse proposée se fonde sur le fonctionnement des systèmes fluviaux en milieu sec et, qui plus est, montagnard (Graf, 1988 ; Bravard et al., 1999). Dans ce cas, le rééquilibrage du système ne suit pas tout à fait la même chronologie qu'en milieu tempéré. En raison de l'altitude et de la latitude, l'accroissement des précipitations dans le bassin-versant du Poulvar ne semble pas s'être traduit immédiatement par une augmentation de la densité de la végétation. D'après N.F. Miller et M. Kimiaie (2004), la reconquête du Zagros par la forêt au Post-Glaciaire se serait faite assez lentement. Ainsi, entre le VIIe et le IVe millénaire avant notre ère, la région du bassin du Kor, dans lequel se jette le Poulvar, comporte une végétation ouverte, basse et faiblement arborée. Les versants seraient restés faiblement couverts, tandis que les précipitations et probablement leur intensité, augmentaient. Ces conditions idéales pour le ruissellement et l'érosion ont sans doute été renforcées par la vigueur des pentes et la nature lœssique des dépôts. Des analyses polliniques supplémentaires restent à faire pour avoir une idée encore plus précise de la nature de la végétation au début de l'OCH. Mais des études menées ailleurs au Proche-Orient ainsi qu'au Maghreb et en Méditerranée, montrent que l'OCH s'accompagne d'une importante sédimentation. C'est ainsi que L. Wengler (1995) décrit l'édification, au Maroc, d'une épaisse nappe alluviale durant l'OCH, dont la phase grossière se dépose lors du passage entre la phase aride et la phase plus humide. En Tunisie, J.-L. Ballais et al. (2003) observent l'édification de terrasses et de cônes alluviaux durant cette période. Le même constat est fait en Syrie, dans les vallées de l'Euphrate et de ses affluents (Besançon et Sanlaville, 1985 ; Geyer et Besançon, 1997). Dans une 
étude sur la péninsule arabique, P. Sanlaville (1992) montre bien que chaque période humide (i.e., pluvial), dont l'OCH, s'accompagne de l'édification d'une terrasse. Enfin, dans leur étude sur l'alluvionnement au sud-est de l'Iran, V. Regard et al. (2006) associent également l'humidité et le phénomène d'érosion-déposition.

L'origine des sédiments constituant la nappe alluviale T1 ne fait pas trop de doute. En revanche, leur épaisseur et leur granulométrie surprennent, dans un milieu peu propice au stockage des particules fines (Ployon, 2006). La vigueur des pentes latérales ainsi que la présence de plaines intramontagnardes à l'aval de la section étudiée ont pu jouer un rôle déterminant. En effet, d'une part, les pentes ont favorisé la fourniture de matériel au cours d'eau ; d'autre part, les plaines successives que le cours d'eau traverse avant de déboucher dans le bassin de Persépolis (fig. 1C), ont contribué à abaisser la pente longitudinale en favorisant une remontée du niveau de base de la vallée. En raison de la diminution du débit à l'aval, il en est résulté une aggradation dans les tronçons amont. Ce phénomène est assez courant dans les régions semi-arides et peut conduire à la formation de profils longitudinaux convexes (Bravard et Petit, 1997). Ainsi, l'érosion des sols et des lœss en amont du bassin-versant a fourni une forte charge solide au cours d'eau dont le débit est resté insuffisant pour évacuer complètement cette charge. Il s'est donc opéré un exhaussement du plancher alluvial.

Ensuite, la nappe alluviale a été très fortement incisée sur une quinzaine de mètres au moins. Cette phase d'érosion, qui constitue un événement majeur dans l'histoire holocène de la région, est encore difficile à dater. Cependant, le niveau sommital de la nappe alluviale $\mathrm{T} 1$, à l'entrée de la plaine de Persépolis, offre une information intéressante. En effet, une coupe stratigraphique, dans les 2,5 m supérieurs de la nappe, montre un niveau sablo-caillouteux hétérométrique à coquilles et charbon de bois sur environ $50 \mathrm{~cm}$. Ce niveau recèle des tessons de céramique émoussés qui, selon les archéologues, ne sont pas antérieurs à 1000 av. J.-C. Une datation radiométrique effectuée sur des charbons de bois présents dans le niveau coquiller a fourni $1758 \pm 45 \mathrm{BP}$. Cet élément de chronologie apporte deux hypothèses différentes sur l'origine du sommet de la nappe alluviale T1 : soit il est formé de simples apports latéraux bien postérieurs au dépôt des alluvions, soit il s'agit de dépôts de crue. Dans le premier cas, cette date ne nous permet pas de s'assurer avec précision de la fin de l'édification de la nappe alluviale ; dans le second cas, la chronologie des événements se voit très fortement resserrée puisque la nappe suivante amorce son édification vraisemblablement un peu avant $1250 \mathrm{BP}$. Ainsi, 400 à 500 ans sépareraient la fin de l'édification de la nappe alluviale supérieure (T1) et l'amorce du dépôt de la nappe alluviale suivante (T2). L'incision de l'épais dépôt T1 aurait lieu sur un temps très court, au regard du temps qu'il a fallu pour sa mise en place. Même si cela n'est pas impossible en milieu aride (Graf, 1988), cette hypothèse nous semble quand même assez peu vraisemblable étant donnée la taille des terrasses. Par ailleurs, le matériel paraît trop grossier pour un dépôt de crue.
L'incision de la nappe T1 s'observe dans l'ensemble du bassin-versant. Il ne s'agit pas d'un phénomène local associé à un événement climatique brutal, tel que cela s'observe souvent en milieu aride ou semi-aride. Il s'agit d'un retournement de l'activité morphologique dans lequel on passe d'un bilan de dissection négatif à un bilan de dissection positif. Ce mouvement peut être consécutif à une forte diminution des apports solides au cours d'eau. Ce phénomène a deux explications principales : soit l'érosion diminue sur les versants en raison d'une forte baisse de la pluviosité, soit elle diminue en raison d'un accroissement de la densité du couvert végétal. Dans notre cas, on constate une aridification du climat dans la région du golfe aux alentours de $5000 \mathrm{BP}$ (Regard et al., 2006). Nous supposons donc que l'incision de la nappe alluviale, qui a pu s'amorcer dès cette date, a pour origine la baisse des apports solides au cours d'eau consécutive à la diminution des précipitations. Le débit liquide a baissé mais, le cours d'eau étant pérenne, l'activité érosive est restée possible. Ce scénario propose un début d'incision bien avant 1000 av. J.-C. Or, dans la mesure où le seul repère chronologique renseignant la fin de l'édification de la nappe alluviale T1 se situe autour de 1000 av. J.-C., nous avons encore besoin de dates pour confirmer cette hypothèse et affiner la chronologie. En revanche, si la date de 1000 av. J.-C. pour l'amorce de l'incision était confirmée, il faudrait s'interroger sur le rôle du climat et de l'Homme dans la région à cette époque, au regard de ce qui s'observe en Méditerranée au même moment. En effet, l'espace méditerranéen traverse, à cette période et jusqu'au début de notre ère, une phase d'intense activité morphologique qui se manifeste par la forte érosion des versants et l'accumulation d'épaisses nappes alluviales, dont les causes sont climato-anthropiques (Vita-Finzi, 1969 ; Dufaure, 1984 ; Fouache, 1999). L'incision majeure de la vallée du Poulvar est probablement aussi pour partie le résultat de l'activité néotectonique. Nous n'avons pas relevé de preuves tangibles de cette tendance, en particulier d'éventuelles micro-failles dans les dépôts alluviaux massifs. Cependant, cette hypothèse doit être posée, sachant que le Zagros est au cœur d'une région instable depuis plusieurs millions d'années (Ross et al., 1986). Le Zagros connaît aujourd'hui un raccourcissement N-S de 7$10 \mathrm{~mm} / \mathrm{a}$ (Tatar et al., 2002 ; Vernant, 2003) qui se traduit par un exhaussement. Sur les 5-6 derniers millions d'années, une vitesse moyenne de raccourcissement similaire a été évaluée (Blanc et al., 2003). Ce phénomène a pu également avoir lieu durant l'Holocène. D'ailleurs, une évaluation ancienne (Mann et Vita-Finzi, 1982) indiquait un raccourcissement de $29 \mathrm{~mm} / \mathrm{a}$ pour cette période. Selon P. Vernant (2003), cette évaluation est surestimée mais la tendance reste vraie. Enfin, de nombreuses autres études indiquent une néotectonique active dans la région du Golfe à l'Holocène (Ross et al., 1986 ; Vita-Finzi, 1986 ; Baltzer et Purser, 1990 ; Potts, 1990 ; Roaf, 1990 ; Pirazzoli, 1991 ; Fontugne et al., 1997 ; Uchupi et al., 1999).

À la suite de cette incision, le retour de l'alluvionnement est dominé par des dépôts grossiers s'achevant par des niveaux fins et une phase de pédogenèse. Les dates obtenues dans cette terrasse T2 montrent que la nappe possède une épaisseur 
d'environ $1 \mathrm{~m}$ vers $1250 \mathrm{BP}$ et de $4 \mathrm{~m}$ vers $1000 \mathrm{BP}$, pour une épaisseur totale d'environ $6 \mathrm{~m}$. Cette sédimentation a plusieurs origines, qui pourraient être en premier lieu l'action des sociétés, sachant que les plaines de Pasargades et de Tang-i Bulaghi sont occupées aux époques pré-islamique et islamique (636 ap. J.-C.), après une présence Achéménide (550-350 av. J.-C.) assez peu marquée. L'occupation et la mise en valeur agricole auraient participé à l'érosion des versants et à la sédimentation dans le Poulvar. Le pastoralisme, qui investit prioritairement les bas de versants, aurait joué un rôle déterminant dans ce phénomène, à une époque où il représente un mode d'occupation des sols fréquent. La responsabilité du pastoralisme et plus généralement de la mise en valeur agricole dans l'érosion des sols sur les versants en pays aride ou méditerranéen est bien connue (Graf, 1988 ; Brückner, 1990 ; Köhler-Rollefson et Rollefson, 1990 ; Baruch, 1994 ; Ballais, 1995 ; Barker, 1995 ; Sanlaville, 1996). Il est possible qu'il faille chercher en second lieu des raisons climatiques à ce retour de l'alluvionnement, mais nous ne disposons pas encore de données paléoécologiques qui pourraient nous renseigner sur la région. En revanche, nous savons que le climat actuel semble s'être établi dès 4500 BP (Stevens et al., 2001). Cependant, des événements climatiques ont pu toucher la région, à l'image du Petit Age Glaciaire (PAG ; Diester-Hass, 1973). Or, un simple changement de l'intensité des précipitations et/ou de leur répartition dans l'année peut déclencher une instabilité morphologique. Par exemple, l'accroissement temporaire des pluies d'automne aurait pu favoriser une érosion des sols au sortir de l'été et accroître la fourniture de matériel au cours d'eau dans un contexte de végétation toujours clairsemée. La nappe alluviale est ensuite incisée sur au moins $6 \mathrm{~m}$. Ce creusement est suivi par une phase de sédimentation récente de faible ampleur, illustrée aujourd'hui par une terrasse de 2-3 m d'épaisseur et datée 14030 BP. Sachant que la moitié supérieure de la terrasse précédente est datée d'environ $1000 \mathrm{BP}$, on peut, avec la prudence nécessaire, situer l'incision au cours du dernier millénaire. Il est difficile, à ce niveau de la recherche, d'argumenter précisément sur ce phénomène qui peut avoir de multiples origines, dont le rééquilibrage du cours d'eau à la suite d'une nouvelle variation du régime des pluies, éventuellement couplé à une migration des populations. En revanche, il est plus aisé d'expliquer la sédimentation grossière qui se met en place ensuite. En effet, la terrasse étant datée 100-200 BP, il est fort probable qu'elle traduise un retour de l'alluvionnement lors de la crise érosive du PAG. Enfin, la période subactuelle a été marquée par l'incision de la nappe que l'on suppose dater du PAG et le façonnement du chenal tel qu'on l'observe aujourd'hui.

\section{Conclusion : la dynamique fluviale caractérisée par une tendance à l'incision depuis la fin de l'OCH}

Les résultats de cette étude constituent des données nouvelles sur une région encore mal connue d'un point de vue de la géomorphologie et du paléoenvironnement. Dans le
Zagros, ils confirment que l'Iran continental connaît un alluvionnement très important à l'Holocène ancien et moyen, à l'image de ce qui s'observe ailleurs en Méditerranée et au Proche-Orient. En apparente contradiction avec le contexte montagnard, l'épaisseur et le faciès des dépôts observés sont expliqués en grande partie par la structure géologique. La rivière Poulvar recoupe par surimposition les plis anticlinaux du Zagros, en particulier sur le tronçon que nous avons étudié. Entre ces anticlinaux, des synclinaux sont aujourd'hui occupés par des plaines comblées de sédiments fins. C'est avant tout la présence de ces plaines d'origine structurale qui a favorisé le dépôt d'une épaisse nappe alluviale limono-sableuse au début de l'Holocène. Le climat a également été un acteur important de la morphogenèse. En effet, l'OCH et surtout la transition entre la période sèche précédente et l'OCH ont favorisé cette dynamique en renforçant l'érosion des sols dans le vaste bassin-versant, sans doute insuffisamment protégés par la végétation au sortir de la dernière période froide.

Les traces postérieures d'activité morphologique, qu'elles concernent les zones de départ du matériel (versants) ou bien les secteurs de transit et d'accumulation (cônes alluviaux, glacis, plaine d'épandage, vallées fluviales) sont modestes. Les vestiges archéologiques observés en subsurface sur les cônes alluviaux de la plaine de Tang-i Bulaghi, dont certains sont datés du Chalcolithique (5 000 av. J.-C.), ou encore les traces de canaux d'âge Achéménide (500-300 av. J.-C.) en place sur les versants du Poulvar, témoignent du ralentissement de l'alluvionnement à partir de l'Holocène moyen. Cette situation est surprenante dans une région où tout devrait concourir à renforcer au contraire cette dynamique : versants raides, contacts brutaux entre les versants et les fonds de vallées, précipitations concentrées, ruissellement, cryoclastie... Le ralentissement de l'alluvionnement ne signifie pas l'absence de morphogenèse. En effet, une phase d'incision majeure a eu lieu après le dépôt de la terrasse supérieure T1. Cet événement, qui reste encore assez mal daté, signale une dynamique très active du cours d'eau dont la charge solide a fortement diminué. Le ralentissement de l'alluvionnement après l'OCH est un phénomène que nous avons pu observer ailleurs au Proche-Orient, dans la steppe syrienne (Rigot, 2006), mais en domaine montagnard, cela est inattendu. Tout se passe comme si, depuis la fin de l'Holocène moyen, en dehors de deux phases de sédimentation historique dont on garde encore aujourd'hui les traces dans la vallée du Poulvar, la dynamique principale aurait été marquée par l'incision linéaire. Cette tendance à l'enfoncement du cours d'eau dans ses propres dépôts n'est pas encore expliquée aujourd'hui par des éléments tangibles. Le climat a probablement joué un rôle déterminant, peut-être associé à une faible mise en valeur, voire même un phénomène de déprise agricole. Mais ces raisons ne sauraient, à elles seules, expliquer ce phénomène. Il faut également rechercher des causes structurales dans cette dynamique et en particulier l'activité néotectonique qui, à travers l'exhaussement du Zagros, a pu être un facteur de contrôle de l'enfoncement à long terme. S'agissant de la sédimentation historique, elle pourrait être en partie d'origine anthro- 
pique, la plaine de Pasargades ayant été occupée en particulier à la période islamique.

\section{Remerciements}

Ce travail a été réalisé dans le cadre d'une collaboration avec la mission archéologique franco-iranienne de Shiraz et sous l'égide du centre de recherche Persépolis-Pasargades. Je me permets de remercier leurs responsables et tout particulièrement R. Boucharlat (MOM, Lyon) qui m'a proposé cette collaboration. Que les relecteurs soient également chaleureusement remerciés et en particulier A. Weisrock et G. Arnaud-Fassetta pour leur lecture attentive et leurs commentaires constructifs ayant permis d'améliorer ce texte. Merci enfin à E. Willcox pour la relecture et les corrections de l'anglais.

\section{Références}

Atai M.T., Boucharlat R. (2009) - An Achaemenid pavilion and other remains in Tang-i Bulaghi. ARTA, 005 (http://www.achemenet.com/document/2009.005-Atai\&Boucharlat.pdf).

Ballais J.-L. (1995) - Conquête et dégradation du milieu au Maghreb oriental pendant l'Antiquité et le Moyen Âge. In Van der Leeuw S. (Ed.) : L'homme et la dégradation de l'environnement. $X V^{e}$ rencontres internationales d'archéologie et d'histoire d'Antibes. Edition APDCA, Sophia Antipolis, 331-344.

Ballais J.-L., Benazzouz M.-T., Benmohamadi A. (2003) Environmental dynamics and land occupation in the Saharan margin of the Holocene Maghreb. In E. Fouache (Ed.): The Mediterranean World Environment and History. Paris, Elsevier, 341-351.

Baltzer F., Purser B.H. (1990) - Modern alluvial fan and deltaic sedimentation in a foreland tectonic setting: the lower Mesopotamian plain and the Arabian Gulf. Sedimentary Geology 76, 175-197.

Barker G. (1995) - Land use and environmental degradation in the Biferno Valley (Central Southern Italy) from Prehistoric times to the Present day. In Van der Leeuw S. (Ed.) : L'homme et la dégradation de l'environnement. XVe rencontres internationales d'archéologie et d'histoire d'Antibes. Edition APDCA, Sophia Antipolis, 285-297.

Baruch U. (1994) - The Late Quaternary pollen record of the Near East. In Bar-Yosef O., Kra R.S. (Ed.): Late Quaternary Chronology and Paleoclimates of the Eastern Mediterranean. Radiocarbon, The University of Arizona-Harvard University, TucsonCambridge, 103-119.

Besançon J., Sanlaville P. (1985) - Résultats de deux prospections archéologiques effectuées dans la région du Nahr Sajour et sur le haut Euphrate syrien. In Sanlaville P. (Ed.) : Holocene Settlement in North Syria. BAR International Series, Oxford, British Archaeological Reports, 7-40.

Bkhairi A., Karray M.-R. (2008) - Les terrasses historiques du basin de Kasserine (Tunisie centrale). Géomorphologie : relief, processus, environnement 3, 201-214.

Blanc E.J.-P., Allen M.B., Inger S., Hassani H. (2003) - Structural styles in the Zagros Simple Folded Zone, Iran, Journal of the Geological Society of London 160, 401-412.
Blanchet G., Sanlaville P., Traboulsi M. (1998) - Le Moyen Orient de 2000 ans à 6000 ans. Essai de reconstitution paléoclimatique. Paléorient, 23, 2, 187-196.

Bobek H. (1963) - Nature and implications of Quaternary climatic changes in Iran. Changes of Climate 20, 403-413.

Boucharlat R., Benech T. (2002) - Organisation et aménagement de l'espace à Pasargades. ARTA, 001 (http://www.achemenet.com/ressources/enligne/arta/pdf/2002.001-loc.pdf).

Bravard J.-P., Petit F. (1997) - Les cours d'eau. Armand Colin, Paris, $222 \mathrm{p}$.

Bravard J.-P., Cammas C., Nehlig G., Poupet P., Salvador P.G., Wattez J. (1999) - La géologie, les sciences de la terre. Errance, Paris, 169 p.

Brückner H. (1990) - Changes in the Mediterranean ecosystem during Antiquity. A geomorphological Approach as Seen in Two Examples. In Bottema S., Entjes-Neiborg G., van Zeist W. (Ed.): Man's Role In The Shaping Of The Eastern Mediterranean. Proceedings of the INQUA/BAI, Rotterdam., A.A. Balkema, 127-137.

Coque R. (1998) - Géomorphologie, Paris, A. Colin, 502 p.

Courty M.-A. (1994) - Le cadre Paléogéographique des occupations humaines dans le bassin du Haut-Khabour (Syrie du Nord-Est). Premiers résultats. Paléorient, 20, 21-59.

Diester-Haass L. (1973) - Holocene climate in the Persian Gulf as deduced from grain-size and pteropod distribution. Marine Geology 14, 207-223.

Djamali M., Soulié-Märsche I., Esu D., Gliozzi E., Okhravi R. (2006) - Palaeoenvironment of a Late Quaternary lacustrine-palustrine carbonate complex: Zarand Basin, Saveh, central Iran. Palaeogeography, Palaeoclimatology, Palaeoecology 237, 315- 334.

Dufaure J.-J. (Ed.) (1984) - La mobilité des paysages méditerranéens. Hommage à Pierre Birot. Revue géographique des Pyrénées et du Sud-Ouest, Toulouse, 387 p.

Erhart H. (1956) - La genèse des sols en tant que phénomène géologique : esquisse d'une théorie géologique et géochimique, biostasie et rhexistasie. Masson, Paris, $90 \mathrm{p}$.

Fisher W.B. (Ed.) (1968) - The Cambridge history of Iran. Volume I, The land of Iran. Cambridge University Press, Cambridge, $784 \mathrm{p}$.

Fouache E. (1999) - L'Alluvionnement historique en Grèce occidentale et au Péloponnèse : géomorphologie, archéologie, histoire. Athènes. Bulletin de correspondance hellénique, 35, 235 p.

Fouache E. (2007) - Dynamiques géomorphologiques holocènes et contexte d'implantation des sites de l'âge du bronze dans la vallée de l'Halil Roud (bassin de Jiroft, SE de l'Iran). Bulletin de l'Association de Géographes Français, 84-2, 132-147.

Fontugne M., Reyss J.L., Hatté C, Pirazzoli P.A., Haghipour A. (1997) - Global sea level changes as indicated by ${ }^{14} \mathrm{C}$ and ${ }^{230} \mathrm{Thr} / 234 \mathrm{U}$ dating of marine terraces in the Persian Gulf and along the Makran coast,Iran. In Meco J., Petit-Maire N. (Ed.): Climates of the Past. Proceedings of the CLIP Meeting, June 27, 1995, Lanzarote and Fuerteventura, Canary Islands, Spain. International Union of Geological Sciences, UNESCO, Earth Processes in Global Change, Universidad de Las Palmas de Gran Canaria, Servicio de Publicaciones, 79-87.

Frey W., Kürschner H. (1989) - Die Vegetation im Vorderen Orient. Erläuterung zur Karte A VI 1 Vorderer Orient. Vegetation 
des « Tübinger Atlas des Vorderen Orients » Wiesbaden, L. Reichert, $92 \mathrm{p}$.

Ganji M.H. (1978) - Post-glacial climatic changes on the Iranian plateau. In Brice W.C. (Ed.): The Environmental History of the Near and Middle East Since the Last Ice Age. Academic Press, London, New York, San Francisco, 149-163.

Geyer B., Besançon J. (1997) - Environnement et occupation du sol dans la vallée de l'Euphrate syrien durant le Néolithique et le Chalcolithique. Paléorient, 22, 5-15.

Graf W.L. (1988) - Fluvial processes in dryland rivers. SpringerVerlag, $346 \mathrm{p}$.

Helwing B., Seyedin M. (2009) - The Achaemenid period occupation at Tang-i Bulaghi site 73. ATAR, 006 (http://www.achemenet.com /document/2009.006-Helwing\&Seyedin.pdf).

Kehl M., Frechen M., Skowronek A. (2005) - Paleosoils derived from loess and loess-like sediments in the Basin of Persepolis, Southern Iran. Quaternary International 140-141, 135-149.

Kehl M., Frechen M., Skowronek A. (2009) - Nature and age of Late Quaternary basin fill deposits in the Basin of Persepolis, Southern Iran. Quaternary International 196, 57-70.

Köhler-Rollefson I., Rollefson G.O. (1990) - The impact of Neolithic subsistence strategies on the environment: the case of Aïn Ghazal, Jordan. In Bottema S., Entjes-Neiborg G., Van Zeist W. (Ed.): Man's Role in the Shaping of the Eastern Mediterranean. Proceedings of the INQUA/BAI, A.A. Balkema, Rotterdam, 3-14.

Kortum G. (1976) - Die Marvdasht-Ebene in Fars : Grundlagen und Entwicklung einer alten iranischen Bewässerungslandschaft Kieler Geographische Schriften, 44. Geographisch Institut der Universität, Kiel, 297 p.

Mann C.D., Vita-Finzi C. (1982) - Curve interpolation and folded strata. Tectonophysics 88, 7-15.

Miller N.F., Kimiaie M. (2006) - Some plants remains from the 2004 excavations of Tall-e Mushki, Tall-e Jari A and B, and Talle Bakun A and B. In Ailzadeh A. (Ed.): The Origins of State Organizations in Prehistoric Highland Fars, Southern Iran: Excavations at Tall-e Bakun. Oriental Institute of the University of Chicago, Chicago, XLIV, 107-118.

Molinaro M. (2004) - Geometry and kinematics of the SE Zagros Mountains (Iran): structural geology and geophysical modeling in a young collisional fold-thrust belt. Thèse en Sciences de la Terre et de l'Environnement, université de Cergy Pontoise, $264 \mathrm{p}$.

Neboit R. (1991) - L'homme et l'érosion : l'érosion des sols dans le monde. Publications de la faculté des Lettres, université Blaise-Pascal, Clermont-Ferrand, 269 p.

Oberlander T.M. (1985) - Origin of drainage transverse to structures in orogens. In Morisawa M., Hack J.T. (Ed.): Tectonic Geomorphology. Allen \& Unwin, Boston-London, 155-182.

Pirazzoli P.A. (1991) - World Atlas of Holocene Sea-Level Changes. Elsevier Oceanography Series, New York, 58, 300 p.

Ployon E. (2006) - Détermination des potentialités d'archivage sédimentaire dans les systèmes fluviaux de montagne : approche morphologique. In Allée P., Lespez L. (Ed.) : L'érosion entre société, climat et paléoenvironnement. Table ronde en l'honneur de R. Neboit-Guilhot. Presses universitaires Blaise Pascal, Clermont-Ferrand, 121-126.
Potts D.T. (1990) - The Arabian Gulf in antiquity. From Prehistory to the Fall of the Achaemenid Empire. Clarendon Press, Oxford, 1, $419 \mathrm{p}$.

Regard V. (2003) - Variations temporelle et spatiale de la transition subduction-collision. Tectonique de la transition ZagrosMakran (Iran) et modélisation analogique. Thèse, université Aix-Marseille 3, 346 p.

Regard V., Bellier O., Braucher R., Gasse F., Bourlès D., Mercier J., Thomas J.-C., Abbassi M.R., Shabanian E., Soleymani S. (2006) - ${ }^{10} \mathrm{Be}$ dating of alluvial deposits from Southeastern Iran (the Hormoz Strait area). Palaeogeography, Palaeoclimatology, Palaeoecology 242, 36-53.

Rigot J.-B. (2003) - Environnement naturel et occupation du sol dans le bassin-versant du lac Jabbûl (Syrie du nord) à l'Holocè$n e$. Thèse de géographie, université Lumière-Lyon 2, 638 p.

Rigot J.-B. (2006) - L'évolution ralentie du milieu naturel dans la steppe aride du nord de la Syrie à l'Holocène. Géomorphologie : relief, processus, environnement 4, 259-274.

Roaf M. (1990) - A cultural atlas of Mesopotamia and the ancient Near East. Equinox, Oxford, 238 p.

Roberts N. (1989) - The Holocene: an environmental history. Oxford, Blackwell, 227 p.

Ross D.A., Uchupi E., White R.S. (1986) - The geology of the Persian Gulf-Gulf of Oman region: a synthesis. Reviews of Geophysics 24, 537-556.

Rossignol-Strick M. (1999) - The Holocene climatic optimum and pollen records of sapropel 1 in Eastern Mediterranean, 90006000 BP. Quaternary Science Reviews 18, 515-530.

Sanlaville P. (1992) - Changements climatiques dans la péninsule arabique durant le Pléistocène supérieur et l'Holocène. Paléorient, 18-1, 5-26.

Sanlaville P. (1996) - Changements climatiques dans la région levantine à la fin du Pléistocène supérieur et au début de l'Holocène. Leur relation avec l'évolution des sociétés humaines. Paléorient, 22-1, 7-30.

Sanlaville P. (1997) - Les changements dans l'environnement au Moyen-Orient de 20000 à 6000 BP. Paléorient, 23, 249-262.

Stevens L.R., Wright Jr. H.E., Ito E. (2001) - Proposed changes in seasonality of climate during the Late glacial and Holocene at Lake Zeribar, Iran. The Holocene 11, 747-755.

Street-Perrot F.A., Roberts N., Metcalfe S. (1985) - Geomorphic implications of Late Quaternary hydrological and climatic changes in the Northern Hemisphere tropics. In Douglas I., Spencer T. (Ed.): Environmental Change and Tropical Geomorphology. George Allen and Unwin, London, 165-183.

Stronach D. (1978) - Pasargadae. Clarendon Press, Oxford, $326 \mathrm{p}$.

Stuiver M., Reimer P.J., Bard E., Beck J.W., Burr G.S., Hughen K.A., Kromer B., McCormac G., Van der Plucht J., Spurk M. (1998) - INTCAL98 Radiocarbon age calibration, 24000-0 cal. BP. Radiocarbon 40-3, 1041-1083.

Sumner W. (1977) - Early Settlements in Fars Province, Iran. In Levine L.D., Young Jr. T.C. (Ed.): Mountains and Lowlands: Essays in the Archaeology of Greater Mesopotamia. Undena Publication, Malibu, 291-305.

Tatar M., Hatzfeld D., Martinod J., Walpersdorf A., GhaforiAshtiany M., Chéry J. (2002) - The present-day deformation of 
the central Zagros from GPS measurements. Geophysical Research Letters 29-19, 1927.

Tricart J. (1977) - Précis de géomorphologie. Tome 2 : Géomorphologie dynamique générale. Sédes, Paris, 345 p.

Uchupi E., Swift S.A., Ross D.A. (1999) - Late Quaternary stratigraphy, Paleoclimate and neotectonism of the Persian-Arabian/Gulf region. Marine Geology 160, 1-23.

Van Zeist W., Bottema S. (1977) - Palynological investigations in western Iran. Palaeohistoria 19, 19-85.

Van Zeist W., Bottema S. (1991) - Late Quaternary vegetation of the Near East. Beihefte zum Tübinger Atlas des Vorderen Orients (TAVO). Reihe A, Naturwissenschaften, 18. L. Reichert, Wiesbaden, $156 \mathrm{p}$.

Vernant P. (2003) - Cinématique actuelle et dynamique de l'Iran : GPS et modélisation numérique. Thèse, université Montpellier 2, $251 \mathrm{p}$.

Vita-Finzi C. (1969) - Mediterranean valleys. Geological changes in historical times. Cambridge University Press, Cambridge, $140 \mathrm{p}$.
Vita-Finzi C. (1986) - Recent earth movements. Academic Press, New York, $226 \mathrm{p}$.

Wengler L. (1995) - Dégradation ou modification du milieu au Maghreb durant l'Holocène : causes anthropiques ou naturelles ? In Van der Leeuw S. (Ed.) : L'homme et la dégradation de l'environnement. XVe rencontres internationales d'archéologie et d'histoire d'Antibes. Edition APDCA, Sophia Antipolis, 315-330.

Wengler L., Vernet J.-L., Michel P. (1994) - Evénement et chronologie de l'Holocène en milieu continental au Maghreb. Les données du Maroc oriental. Quaternaire, 5, 3-4, 119-134.

Weisrock A., Wahl L., Ouammou A., Chakir L. (2006) - Systèmes fluviaux du Sud-Ouest marocain et leur évolution depuis le Pléistocène supérieur. Géomorphologie : relief, processus, environnement 4, 229-248.

Zohary M. (1973) - Geobotanical foundations of the Middle East. Second volume. G. Fischer Swets \& Zeitlinger, StuttgartAmsterdam, 341-738.

Article soumis le 18 octobre 2007, accepté le 19 décembre 2009. 